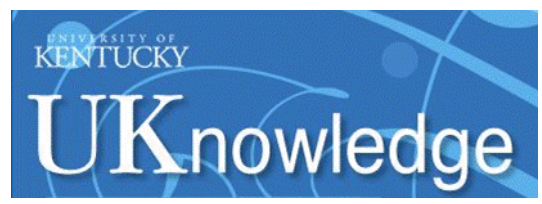

University of Kentucky

UKnowledge

4-20-1996

\title{
High Metal Enrichments in Luminous Quasars
}

\author{
Gary J. Ferland \\ University of Kentucky, gary@uky.edu \\ Jack A. Baldwin \\ Cerro Tololo Inter-American Observatory, Chile \\ Kirk T. Korista \\ University of Kentucky \\ Fred Hamann \\ University of California - San Diego \\ R. F. Carswell \\ Institute of Astronomy, UK
}

See next page for additional authors

Follow this and additional works at: https://uknowledge.uky.edu/physastron_facpub

Part of the Astrophysics and Astronomy Commons, and the Physics Commons

Right click to open a feedback form in a new tab to let us know how this document benefits you.

\section{Repository Citation}

Ferland, Gary J.; Baldwin, Jack A.; Korista, Kirk T.; Hamann, Fred; Carswell, R. F.; Phillips, Mark; Wilkes, Belinda J.; and Williams, Robert E., "High Metal Enrichments in Luminous Quasars" (1996). Physics and Astronomy Faculty Publications. 149.

https://uknowledge.uky.edu/physastron_facpub/149

This Article is brought to you for free and open access by the Physics and Astronomy at UKnowledge. It has been accepted for inclusion in Physics and Astronomy Faculty Publications by an authorized administrator of UKnowledge. For more information, please contact UKnowledge@lsv.uky.edu. 


\section{High Metal Enrichments in Luminous Quasars}

\section{Digital Object Identifier (DOI)}

http://dx.doi.org/10.1086/177094

\section{Notes/Citation Information}

Published in The Astrophysical Journal, v. 461, no. 2, p. 683-697.

(c) 1996. The American Astronomical Society. All rights reserved.

The copyright holder has granted permission for posting the article here.

\section{Authors}

Gary J. Ferland, Jack A. Baldwin, Kirk T. Korista, Fred Hamann, R. F. Carswell, Mark Phillips, Belinda J. Wilkes, and Robert E. Williams 


\title{
HIGH METAL ENRICHMENTS IN LUMINOUS QUASARS
}

\author{
Gary J. Ferland, ${ }^{1,2}{ }^{2}$ ACK A. Baldwin, ${ }^{1}$ KIRK T. Korista, ${ }^{2}$ Fred Hamann, ${ }^{3}$ R. F. Carswell, ${ }^{4}$ \\ Mark Phillips, ${ }^{1}$ Belinda Wilkes, ${ }^{5}$ and Robert E. Williams ${ }^{1,6}$ \\ Received 1995 July 19; accepted 1995 October 27
}

\begin{abstract}
We discuss observations of the broad emission line in luminous intermediate redshift quasars, with emphasis on Q0207-398. This object has relatively sharp lines, allowing us to deconvolve the profiles into distinct components. In this paper we examine the high ionization region, which we show must be matter-bounded, partially transparent to ionizing radiation. We measure a large $\mathrm{N} v \lambda 1240 / \mathrm{He}$ II $\lambda 1640$ ratio in Q0207-398, but detailed calculations predict this ratio to be $\sim 1$ for standard cloud properties. We show that no choice of incident continuum, ionizing photon flux, density, or even the appeal to nonradiative energy sources, can reproduce the observed N v, C IV, O VI, He II spectrum with solar abundances. The intensity of the $\mathrm{N} v$ line is sensitive to the abundance of the gas because the abundance of nitrogen, a secondary element, goes up roughly as the square of the metallicity. The lowest metallicity capable of reproducing the $\mathrm{N} v$ spectrum in $\mathrm{Q} 0207-398$ is $Z \approx 5 Z_{\odot}$, consistent with that inferred to exist in the cores of massive galaxies after epochs of rapid star formation.
\end{abstract}

Our final model of the high-ionization emission region is one with many properties similar to those inferred for broad absorption-line QSO absorption-line clouds.

Subject headings: galaxies: abundances - quasars: emission lines - quasars: individual (Q0207-398)

\section{INTRODUCTION}

Luminous QSOs are generally thought to occur during the very early stages of a galaxy's life. The heavy-element abundances in the broad line region (BLR) can, in principle, be deduced from a QSO's emission-line spectrum. This would allow us to directly observe the details of early galactic nucleosynthesis. The BLR is the gas in the innermost part of the parent galaxy, and whether it is in the process of being accreted onto a black hole or whether it is there for some other reason, it must have been through whatever enrichment processes have occurred in the stellar population in the inner few hundred parsecs of the host galaxy.

Studies of the central regions of massive galaxies reveal high metallicities, $\langle Z\rangle \lesssim 5 Z_{\odot}$, among the population of very old stars, showing that an epoch of vigorous star formation rapidly generated a chemically enriched environment (Arimoto \& Yoshi 1986; Bica 1988; Bica, Arimoto \& Alloin 1988). Nearly as large metallicities are observed among old stars in the bulge of our own Galaxy (a mean of $\langle Z\rangle \approx 1-2 Z_{\odot}$ and a maximum of $Z \approx 10 Z_{\odot}$; Rich 1988; McWilliam \& Rich 1994). Thus the stellar population observations require rapid enrichments at an early epoch. If BLR gas is coupled to the interstellar medium (ISM) of the host galaxy, then knowledge of the BLR metallicity as a function of redshift would map out the enrichment process out to look-back times corresponding to redshifts $z>4$. This is a major long-term goal of quasar research.

Unfortunately, abundance measurements for BLR gas have been elusive. "Standard " BLR models trying to reproduce just the strongest lines cannot tell much about the

\footnotetext{
${ }^{1}$ Cerro Tololo Inter-American Observatory, NOAO, Casilla 603, La Serena, Chile.

${ }_{2}^{2}$ Physics Department, University of Kentucky, Lexington, KY 40506.

${ }^{3}$ Center for Astrophysics and Space Sciences, Univ. of California-San Diego, La Jolla, CA 92093.

${ }^{4}$ Institute of Astronomy, Madingley Road, Cambridge, CB3 0HA, England.

${ }_{5}^{5}$ Smithsonian Astrophysical Observatory, 60 Garden Street, Cambridge, MA 02138 .

6 STScI, 3700 San Martin Dr., Baltimore, MD 21218.
}

heavy-element abundances because the strong lines mainly reflect the balance of heating and cooling in the gas. Shields (1976) suggested using intercombination lines of intermediate stages of ionization of several elements to measure the relative abundances. This approach has not been used widely, mainly because the lines in question are generally too weak to measure, but also because intercombination lines are subject to collisional quenching (see also Uomoto 1984) so that the BLR density must be well understood.

Another approach has been to use the intensity ratio $\mathrm{N} \mathrm{v}$ 1240/C IV 1549 (Hamann \& Ferland 1992, 1993; see also Osmer, Porter, \& Green 1994). These are strong lines that can be measured to at least rough precision in many QSOs. The key to the method is that nitrogen is predicted to be largely a secondary element in the cores of massive galaxies. Thus, it is built up quadratically with $Z$, while carbon, a primary element, is built up only linearly. The N/C abundance ratio should then depend linearly on the $\mathrm{C} / \mathrm{H}$ abundance ratio (which is proportional to the metallicity $Z$ ). In this paper we will show that the $\mathrm{N} v \lambda 1240 / \mathrm{He}$ II $\lambda 1640$ intensity ratio can also be used as an abundance indicator. This ratio has the advantage that $\mathrm{N} \mathrm{V}$ and $\mathrm{He}$ II are generally produced in overlapping regions. Although it is possible to contrive geometries where this ratio underestimates the abundances, we can see no way in which it would produce an overestimate. We shall see that both ratios suggest metallicities $\gtrsim 5 Z_{\odot}$ in the most luminous objects in our sample.

The organization of the paper is to first (§2) establish a fiducial data set of observed emission-line intensity ratios. We will focus heavily on one particular QSO, Q0207-398, which was described in an accompanying paper (Baldwin et al. 1996; hereafter Paper I). Its relatively narrow lines allow us to distinguish kinematically the $\mathrm{N} v$ and $\mathrm{He}$ II emitting gas from the low ionization regions, so that a better dynamical model can be developed. Next (§3) we will show that the very high $\mathrm{N} v / \mathrm{He}$ II ratio $(\mathrm{N} v / \mathrm{He}$ II $>10)$ in Q0207-398 cannot possibly come from gas with solar abundances. This conclusion is independent of the energy 
source of the cloud - neither photoionization nor collisional ionization can reproduce the observed ratio using $Z=Z_{\odot}$. In $\S 4$ we show that the observed intensity ratios require $Z \gtrsim 5 Z_{\odot}$.

We will show that the high-ionization lines must arise in a region which is partially transparent to ionizing radiation, with significant covering factor. Both the level of ionization and the matter-bounded geometry are reminiscent of BALQSO absorption-line regions. Our final best-fitting model is one with a metallicity 5 times solar and a covering factor of $\approx \frac{1}{5}$. The high-ionization region may be related to the outflowing wind predicted to occur during the last stages of the evolution of central regions of massive galaxies.

\section{OBSER VED $\mathrm{N} \mathrm{v} / \mathrm{He}$ II INTENSITY RATIOS}

\subsection{Complete Samples}

We will first define the observed range of $\mathrm{N} \mathrm{v/He} \mathrm{II} \mathrm{inten-}$ sity ratios in the general population of luminous QSOs. We will draw on two well-studied and statistically complete samples of QSOs for which we have digital spectra readily at hand: the PKS \pm 4 deg sample and the Bright Quasar Survey (BQS). Our spectra of these objects appeared in Baldwin, Wampler, \& Gaskell (1989).

The problems in measuring the $\mathrm{N} v / \mathrm{He}$ II ratio are twofold. First, $N$ v is badly blended with the strong Ly $\alpha$ emission line. Second, $\mathrm{He}$ II is always a weak line, and tends to blend with $\mathrm{O}$ III $\lambda 1666$ and other unidentified features to form a broad shelf on the red wing of the strong C IV 1549 emission line. It is therefore frequently impossible to measure one or both of the lines needed to form the $\mathrm{N} \mathrm{v}$ / He II ratio.

Of the 46 high-redshift QSOs listed in Tables 2 and 7 of Baldwin et al. (1989), it is possible to make a useful separation between the Lya and $\mathrm{N} \mathrm{v}$ lines for 14 . Of those 14 objects, $\mathrm{He}$ II is actually distinguishable as a separate line in only four cases; for the other 10 QSOs we can measure only upper limits for the He II strength. The results are given in Table 1, where we also include the strengths of a few other important lines and the absolute continuum magnitudes of the QSOs. All of the line strength measurements were made by scaling the strength and position of the C IV profile to the profile of interest; in these particular objects the different lines appear to have sufficiently similar profiles that this method gives the best results for the noisy, blended line profiles.

We will show below that for solar abundances we expect $\mathrm{N} \mathrm{v} / \mathrm{He}$ II $\lesssim 2$, and that values $\gg 1$ indicate higher than solar heavy-element abundances. Table 1 shows that the $\mathrm{N} \mathrm{v} / \mathrm{He}$ II ratio is typically in the range $3-8$, and that very few QSOs of this type have values as low as 2 . This is to be contrasted with observations of the well-studied Seyfert galaxy NGC 5548, which show a N v/He II ratio consistent with solar abundances (Clavel et al. 1991). Although the QSO measurements have the advantage of coming from samples with well-understood selection effects, the data are in general too noisy and of too low spectral resolution to allow very accurate results.

\subsection{Some Better Measurements}

To get better measurements for a smaller set of objects, we turn to the spectra described in Paper I. These include the spectra of four luminous $z \sim 2-3$ QSOs for which highresolution spectra of the $\mathrm{Ly} \alpha$ forest region were already available in the literature. We began to study these objects because of an interest in detecting or setting limits on the strength of the C III $\lambda 977$ emission line, and a full discussion of all of the emission lines is given in Paper I. Here we concentrate on the regions of gas which form the $N v \lambda 1240$ and $\mathrm{He}$ II $\lambda 1640$ emission lines. In addition to the four very luminous QSOs, we take from Paper I the spectrum of the lower luminosity object Q1451+1017 $[z=2.17$, $\left.M_{\mathrm{v}}(1549)=-24.37\right]$, which because of the very sharp emission lines cores also permits a good measurement of the $\mathrm{N} \mathrm{v} / \mathrm{He}$ II ratio.

We show here (Fig. 1) blow-ups of the spectral regions around $\mathrm{N} v$ and $\mathrm{He}$ II, along with fitted profiles derived from other lines in the spectra. It is clearly possible to obtain either fairly accurate measurements or (in the case of some of the $\mathrm{He}$ II lines) tight upper limits for the line strengths.

Table 2 lists the measured intensity ratios and absolute magnitudes in the same format as Table 1 . Again, most of the objects have $\mathrm{N} \mathrm{v/He} \mathrm{II}>3$. The conspicuous exception is the low-luminosity object Q1451+1017, with $\mathrm{N} \mathrm{v/He} \mathrm{II}$ $\sim 0.5$. We will focus our attention on the two objects at the

TABLE

RESUlTS FOR QSOS FROM Two COMPLETE SAMPLES

\begin{tabular}{|c|c|c|c|c|c|}
\hline Object & $\mathrm{N}$ v/He II & $\mathrm{N}$ v/C IV & $\mathrm{He}$ II/C IV & $W_{\lambda}(\mathrm{N} v)$ & $M_{\mathrm{v}}(1549)$ \\
\hline \multicolumn{6}{|c|}{ Detections of $\mathrm{He}$ II } \\
\hline PKS 0226-038. & 7.07 & 0.99 & 0.14 & 27.5 & -26.65 \\
\hline 3C $273 \ldots \ldots \ldots$ & 3.07 & 0.46 & 0.15 & 12.3 & -26.34 \\
\hline PKS $2134+004 \ldots \ldots$ & 5.00 & 0.80 & 0.16 & 26.2 & -26.94 \\
\hline BQS $1247+267 \ldots \ldots$ & 7.83 & 0.94 & 0.12 & 31.2 & -28.48 \\
\hline \multicolumn{6}{|c|}{ Upper limits on $\mathrm{He}$ II } \\
\hline PKS $0421+019$. & $>1.97$ & 0.59 & $<0.30$ & 10.1 & -26.89 \\
\hline PKS $0505+03 \ldots \ldots$ & $>1.92$ & 0.48 & $<0.25$ & 9.2 & -26.25 \\
\hline PKS $0922+005 \ldots \ldots$ & $>5.35$ & 1.66 & $<0.31$ & 44.8 & -25.99 \\
\hline PKS $1402-012 \ldots \ldots$ & $>0.68$ & 0.28 & $<0.41$ & 12.8 & -26.28 \\
\hline PKS $2254+024 \ldots \ldots$ & $>2.57$ & 0.59 & $<0.23$ & 17.1 & -26.30 \\
\hline BQS $1138+040 \ldots \ldots$ & $>3.16$ & 1.58 & $<0.50$ & 36.5 & -27.00 \\
\hline BQS $1222+228 \ldots \ldots$ & $>4.62$ & 1.34 & $<0.29$ & 36.0 & -27.77 \\
\hline BOS $1329+412 \ldots \ldots$ & $>2.47$ & 0.47 & $<0.19$ & 27.3 & -27.05 \\
\hline BQS $1715+535 \ldots \ldots$ & $>6.24$ & 1.56 & $<0.25$ & 25.7 & -27.84 \\
\hline BQS $1115+080 \ldots \ldots$ & $>3.00$ & 0.81 & $<0.27$ & 25.8 & -27.78 \\
\hline
\end{tabular}




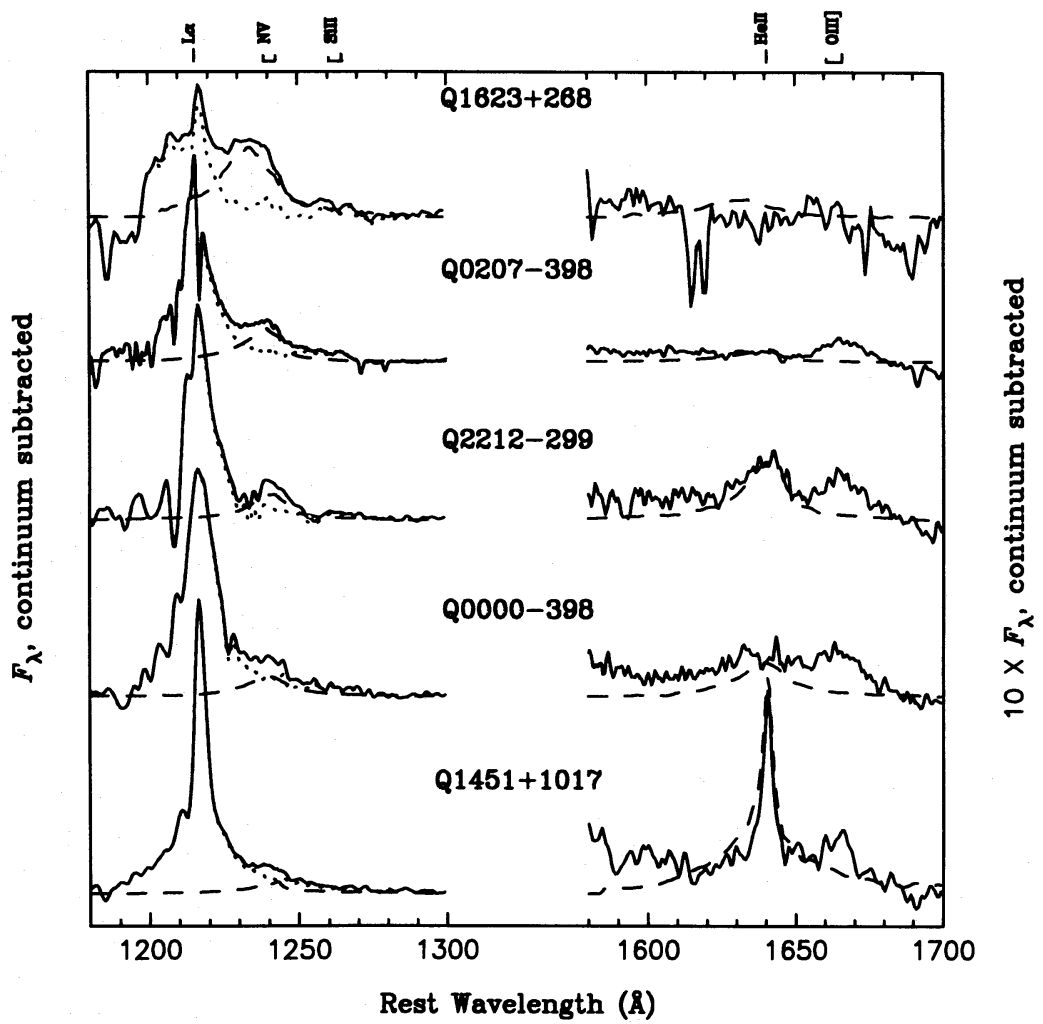

FIG. 1.-The regions around N v $\lambda 1240$ (left) and He II $\lambda 1640$ (right) for five well-observed QSOs. For each spectrum, the underlying continuum has been subtracted off and the right plot is blown up to a scale 10 times that of the left one. The dashed lines show the $\mathrm{N} v$ fits and the He II fits or upper limits, with the strengths from Table 2 . The dotted line is the residual.

opposite end of the scale: Q1623 + 268, with N v/He II > 31, and $\mathrm{Q} 0207-398$, with $\mathrm{N}$ v/He II $>21$. These extremely high $\mathrm{N} \mathrm{V} / \mathrm{He}$ II ratios place the hardest constraints on attempts to model the BLR gas with nearly-solar abundances, and therefore will be the most important for testing our idea that at least some QSOs have high metal abundances in their BLRS. In addition, for both of these QSOs the $\mathrm{N} v$ and $\mathrm{C}$ IV lines seem to have a profile which is different from those of lower ionization lines such as Ly $\alpha$ and C III] 21909 . These profile differences give us a start at sorting out the BLR gas into different kinematic zones which are presumably physically distinct, so that we can identify the gas which contributes very high ionization emission lines such as $\mathrm{N} v$ and $\mathrm{He}$ II. In both of these objects we have only an upper limit on the He II strength, but it is a tight upper limit in combination with the clear presence of strong $\mathrm{N} \mathrm{v}$ emission. In fact, comparison of the $\mathrm{N} \mathrm{v/C} \mathrm{IV}$ and $\mathrm{He} \mathrm{II} / \mathrm{C}$ IV ratios to those of the other objects in Tables 1 and 2 shows that in these two QSOs He II is unusually weak at the same time that $\mathrm{N} v$ is unusually strong.

\subsection{Q0207-398}

This is the particular object to which we will attempt to fit most of our models throughout the remainder of this paper. This is both because it is one of the two objects noted above as having an especially high $\mathrm{N} \mathrm{v} / \mathrm{He}$ II ratio, and also because it has the spectrum in which we can most clearly separate the different BLR zones. There are at least three kinematically distinct zones, which we call components A, $\mathrm{B}$, and $\mathrm{C}$ (see Fig. 2). The $\mathrm{C}$ IV and $\mathrm{N} v$ emission lines have FWHM $=3900 \mathrm{~km} \mathrm{~s}^{-1}$ and asymmetric blue tails (component B); this profile is easily distinguished from those of Ly $\alpha\left(\mathrm{FWHM}=2000 \mathrm{~km} \mathrm{~s}^{-1}\right.$; component C) and lines such as Al III 1860 (1000 $\mathrm{km} \mathrm{s}^{-1}$ FWHM; component A).

Paper I discusses how we have separated the emission lines between the different BLR components and presents our interpretation of the narrower lined region. The basic results are that we can make reasonably accurate measurements of the strengths of $\mathrm{N} \mathrm{v}$ and $\mathrm{C}$ IV, despite the blending

TABLE 2

Results For Five Well-ObSERVED QSOS

\begin{tabular}{cccccc}
\hline \hline Quasar & N v/He II & N v/C IV & He II $/ C$ IV & $W_{\lambda}(\mathrm{N}$ v $)$ & $M_{\mathrm{v}}(1549)$ \\
\hline Q1451 $+1017 \ldots \ldots$ & 0.6 & $\leq 0.16$ & 0.25 & 9.2 & -24.37 \\
Q0000-398 $\ldots \ldots .$. & 4.7 & 0.31 & $\leq 0.07$ & 16.4 & -26.12 \\
Q2212 $-299 \ldots \ldots$. & 3.6 & 0.58 & 0.16 & 10.6 & -27.32 \\
Q1623 $+268 \ldots \ldots$. & $>31$. & 1.9 & $<0.06$ & 15.3 & -27.36 \\
Q0207-398 $\ldots \ldots$. & $>21$. & 1.1 & $<0.05$ & 19.8 & -27.37 \\
\hline
\end{tabular}

a The entries for Q0207-398 are total intensities, summed over all three components. Table 4 of Paper 1 shows how we deconvolve this sum into kinematic components. 


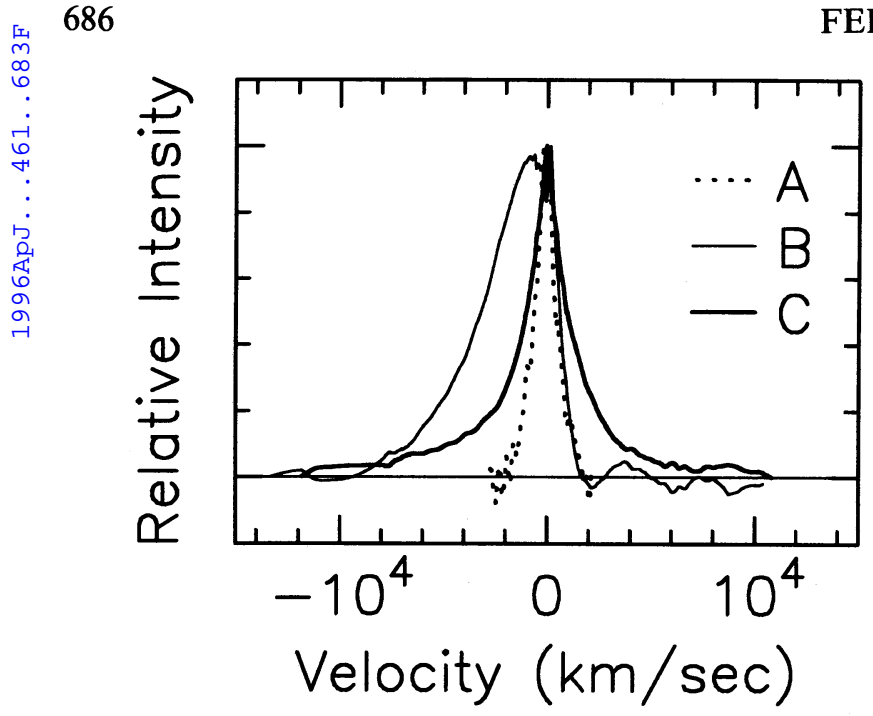

FIG. 2.-The different velocity components in the emission-line profiles of Q0207-398.

of $\mathrm{N} \mathrm{v}$ with the Ly $\alpha$ line. The other high-ionization line which we can measure with some degree of accuracy is $\mathrm{O}$ VI 1034, although there is some doubt about the profile fit because this area of the spectrum is chopped up by Ly $\alpha$ forest absorption. The fit of component $B$ to this doublet is shown in Figure 3.

None of the other emission lines appears to have a profile which matches that of component B, and so in Paper I we assigned no component $B$ flux to those lines. However, the profile of component $\mathbf{C}$ (the one with intermediate width) could in fact be a combination of some arbitrary mixture of the component B profile plus some additional profile which is not shown in Figure 2. The upper limit on the possible component $\mathrm{B}$ contribution to these other lines is the flux at which a component B profile starts to poke above the top of the actual observed profile (55\% of the flux for the case of a pure component $\mathbf{C}$ profile). It is this sort of limit which we use in the following analysis.

Table 3 lists measured fluxes and upper limits for each line, relative to $\mathrm{N} \mathrm{v}$, and also the rest frame equivalent width of the fraction of $\mathrm{N} \mathrm{v}$ which we assign to component

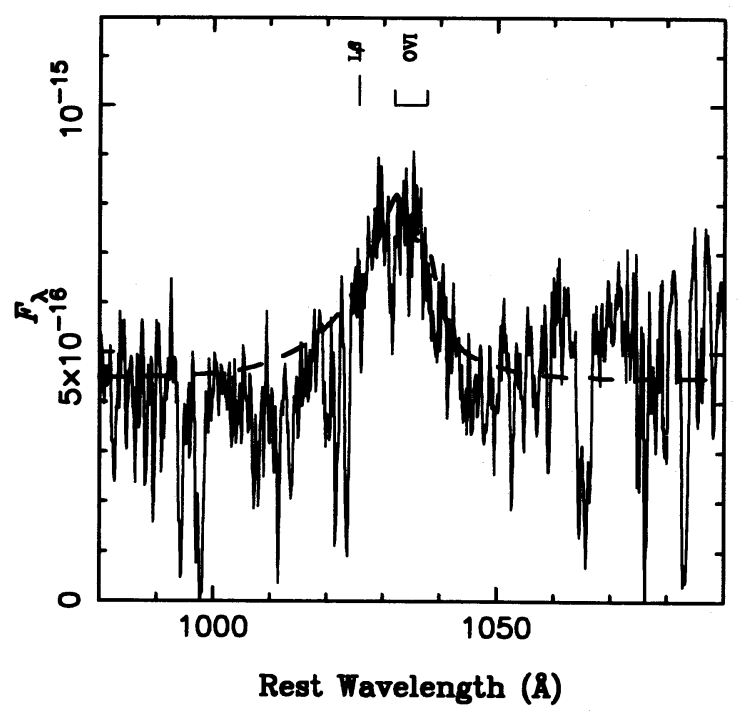

Fig. 3.-The fit of component B to the O vi $\lambda \lambda 1031.95,1037.63$ doublet in the spectrum of Q0207-398. The two lines are assumed to be optically thick and therefore of equal intensity.
TABLE 3

COMPONENT B OF Q0207-398

\begin{tabular}{|c|c|c|}
\hline $\begin{array}{l}\text { Line } \\
\text { (1) }\end{array}$ & $\begin{array}{c}\text { Best } \\
\text { Estimate of } \\
\text { Intensity }^{\mathrm{a}} \\
(2)\end{array}$ & $\begin{array}{c}\text { Weakest } \\
\text { Possible N v } \\
\text { (3) }\end{array}$ \\
\hline S VI $933 \ldots \ldots \ldots \ldots$ & $<0.25$ & $<0.55$ \\
\hline $\mathrm{C}_{\text {IIII }} 977+\operatorname{Ly} \gamma \ldots \ldots$ & $<0.22$ & $<0.48$ \\
\hline $\mathrm{N}$ III $990 \ldots \ldots \ldots \ldots$ & $<0.22$ & $<0.48$ \\
\hline O vi $1035 \ldots \ldots \ldots \ldots$ & 0.95 & $<2.09$ \\
\hline Ly $\alpha 1216 \ldots \ldots \ldots \ldots$ & $<2.55$ & $<5.62$ \\
\hline $\mathrm{N} v 1240 \ldots \ldots \ldots \ldots$ & 1.00 & 1.00 \\
\hline \multicolumn{3}{|l|}{ Si IV $1397+$} \\
\hline O Iv] $1402 \ldots \ldots \ldots \ldots$ & $<0.23$ & $<048$ \\
\hline N IV $] 1486 \ldots \ldots \ldots$ & $<0.054$ & $<0.12$ \\
\hline C IV $1549 \ldots \ldots \ldots \ldots$ & 0.90 & $<1.98$ \\
\hline He II $1640 \ldots \ldots \ldots$. & $<0.11$ & $<0.24$ \\
\hline O III] $1666 \ldots \ldots \ldots$. & $<0.09$ & $<0.20$ \\
\hline Al III $1860 \ldots \ldots \ldots$. & $<0.018$ & $<0.040$ \\
\hline Si III $] 1895 \ldots \ldots \ldots \ldots$ & $<0.018$ & $<0.040$ \\
\hline C III] $1909 \ldots \ldots \ldots$ & $<0.14$ & $<0.31$ \\
\hline$W_{\lambda}(\vec{N} v)^{b} \ldots \ldots \ldots \ldots$ & $9.7 \AA$ & $4.4 \AA$ \\
\hline
\end{tabular}

a Intensity relative to $\mathrm{N} v 1240$, for the $\mathrm{N} v$-emitting region.

${ }^{b}$ Equivalent width of component B N v. Component $\mathrm{C}$ emits a similar amount, and the total observed equivalent width is $19.8 \AA$.

B. The column entitled "best estimate" is based on fitting the component B profiles. We take this as the set of observed parameters to which we will fit our models.

Finally, to assess the uncertainties due to $\mathrm{N} v$ being badly blended with $\mathrm{Ly} \alpha$, we defined a weakest possible $\mathrm{N} v$ profile by just subtracting off the highest possible smooth Ly $\alpha$ wing (Fig. 4). The residual profile, which has only about one-half as much flux as the component $\mathrm{B}$ fit to $\mathrm{N} \mathrm{v}$, does not fit the profile of any other line in the spectrum. However, we went ahead and found upper limits for how much of this new profile could fit under each of the lines listed in Table 3. Those values are listed in the column of Table 3 entitled "Weakest Possible N v," and give an idea of the extreme error bars which should be assigned to each of these measurements. We do not use these values further since this interpretation results in a line profile for $\mathrm{N} v$ which is unlike

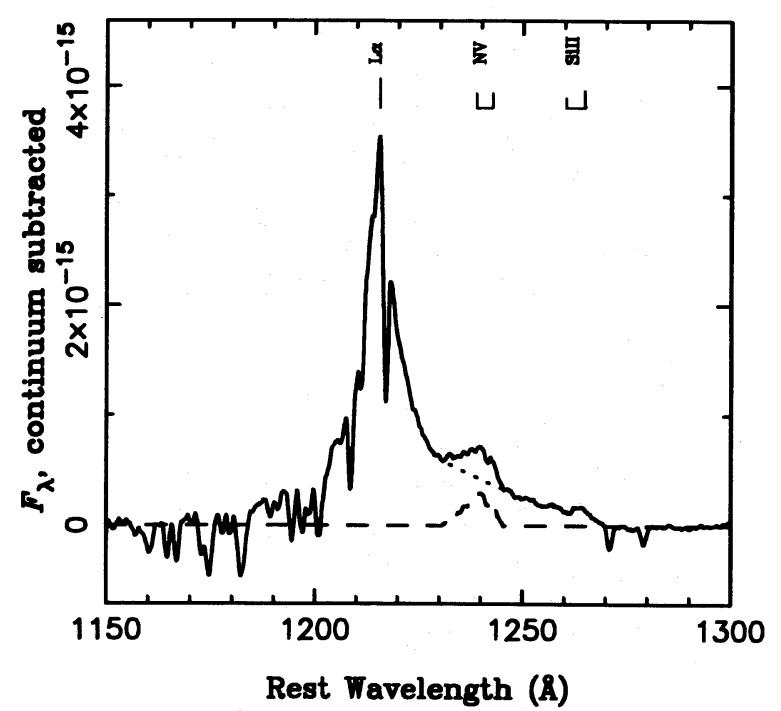

FIG. 4. - The $\mathrm{N} v \lambda 1240$ region in the spectrum of $Q 0207-398$ showing the fit used to derive the profile of the weakest possible $\mathrm{N} v$ line (dashed line $=\mathrm{N} v$ profile; dotted line $=$ residual Ly $\alpha$ wing). 
any of the other observed profiles. Note that changing the assumed line profile by even this much has little effect on the deduced limit to $\mathrm{N} \mathrm{v} / \mathrm{He}$ II.

In all this, we stress that our approach of employing line component deconvolution does not alter the deduced emission-line spectrum by very much. In this paper we shall concentrate on the $\mathrm{N} v / \mathrm{He}$ II ratio of component $\mathrm{B}$. The observed total line ratio is $\mathrm{N} \mathrm{v/He} \mathrm{II} \geq 21$ while the component $\mathrm{B}$ ratio is $\geq 10$. This is a conservative limit to the line ratio, and will lower our deduced metallicity by roughly a factor of 2 .

\section{PHOTOIONIZATION MODELS}

\subsection{An Analytical Form for the Continuum}

The continuum must be fully specified between very low energies (important sources of free-free-heating or Compton cooling), and very high energies (important for photoelectric and Compton heating). The overall shape striking the BLR may or may not be similar to that actually observed. This shape is described by a "big bump," likely to be of thermal origin. This big bump is thought to be due to reprocessing of some kind (Guilbert \& Rees 1988; Barvainis 1990, 1992; Rokaki \& Magnan 1992; Rokaki, Collin-Souffrin, \& Magnan 1993) or to an accretion disk (Malkan \& Sargent 1981; Laor \& Netzer 1989). The nonthermal power-law $\mathrm{X}$-ray continuum has been reflected off a "Compton reflector"(Lightman \& White 1988; Begelman 1989).

It now seems likely that the near-infrared (longward of $1 \mu \mathrm{m})$ portion of the observed continuum is formed by dust emission outside the BLR radius (Sanders et al. 1989; Barvainis 1990,1992$)$. As a result the intensity of the nearinfrared continuum at the BLR radius is essentially unkown, but is crucial since free-free heating by a nonthermal continuum could dominate the heating at the illuminated face of a cloud and can lead to a thermal runaway, in which the gas changes phase from the warm to hot phase (Krolik, McKee, \& Tarter 1981; Ferland et al. 1992). Thus not only does a cloud's spectrum depend on the IR continuum, but the cloud's very existence does as well.

We have taken the approach of adopting the simplest continuum consistent with the observations outlined above. For reference Figure 5 shows a typical continuum used in the calculations. The soft component is the rising (in $v F_{v}$ ) UV "big bump," which is approximated as a power law with exponential cutoffs at both high and low energies (see also Mathews \& Ferland 1987; Binette et al. 1989; Kallman et al. 1993). This choice is motivated by observations such as those described by Malkan \& Sargent (1982) and Wilkes $\&$ Elvis (1987). We generated models with $f_{v} \propto v^{-0.5}$ and $f_{v} \propto v^{+0.5}$, but in the final models we assume a power law, $f_{v} \propto v^{0}$ since this, when combined with the exponential cutoff described below, most closely fits the observed continuum. Although the details did change, the major results presented below did not depend on the slope. Both ends of this continuum source were cut off exponentially. The lowenergy cutoff of $1000 \mathrm{~K}$ was chosen to be low enough for a significant infrared continuum to come from this continuum source, but yet high enough to prevent unrealistically high IR brightness temperatures. The high-energy cutoff was varied, as described below. The full form of the "big bump" continuum is thus given by

$$
f_{v}(\text { Big Bump }) \propto v^{0} \exp \left(-h v / k T_{\text {cutoff }}\right) \exp \left(-k T_{\text {Iow }} / h v\right),
$$

where $T_{\text {low }}$ and $T_{\text {cutoff }}$ are the low and high-energy cutoffs. In much of the following we shall refer to this component as the soft component. The high-energy cutoff $T_{\text {cutoff }}$ has a great impact on the intensities of the BLR spectrum, and its effect will be described further below. Tests show that the choice of the low-energy cutoff has little effect on the spectrum as long as the density is low enough to minimize the free-free heating (i.e., $n_{\mathrm{H}} \leq 10^{12} \mathrm{~cm}^{-3}$ ). It is quite important for the denser clouds we consider below, however.

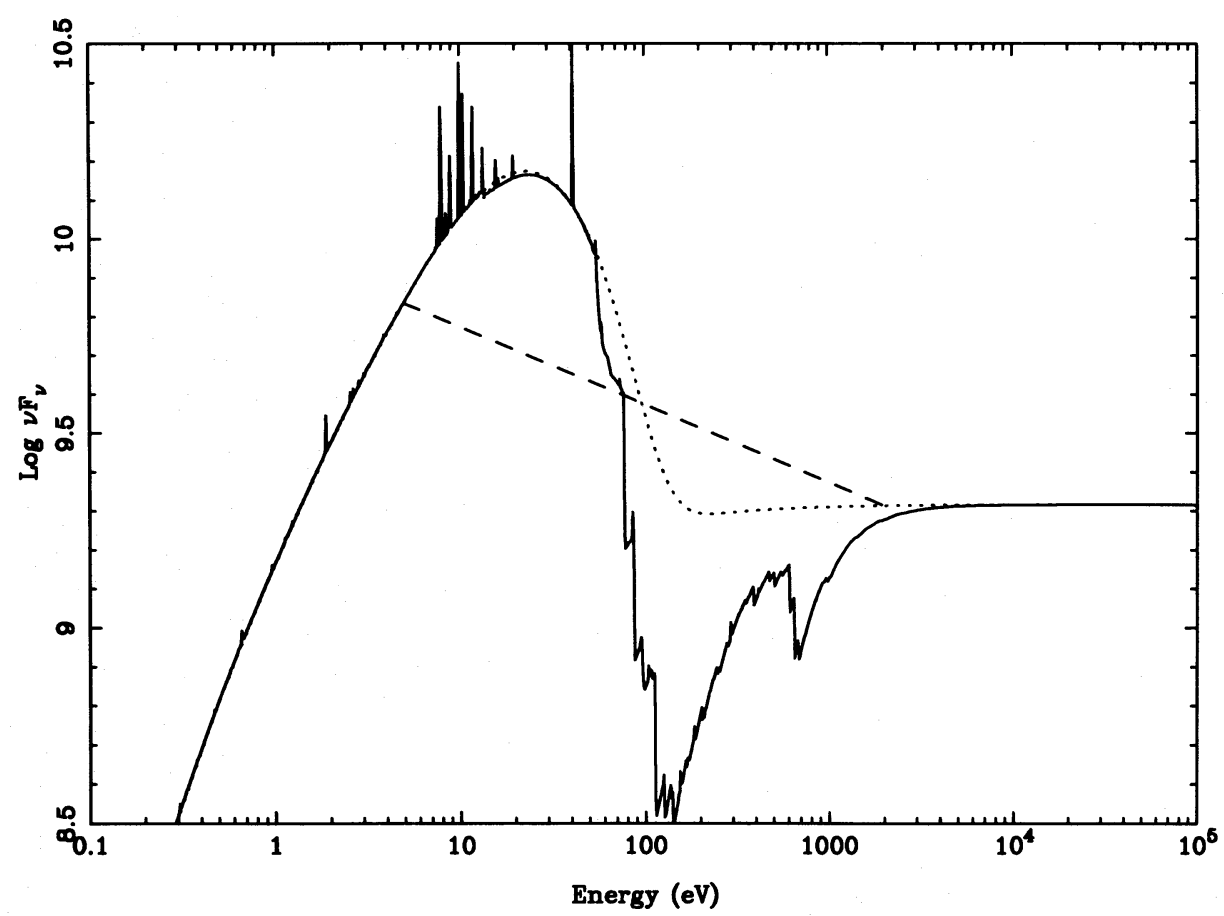

Fig. 5.-The transmitted continuum for the case of full coverage of the continuum source by absorbing gas is shown as a solid line. The dotted curve is the incident continuum. The dashed line indicates $\alpha_{o x}$. 
The second component is a high-energy power-law continuum extending into the hard $\mathrm{X}$-ray energies. For this hard component we adopt a power-law continuum with slope -1 , i.e., $f_{v} \propto v^{-1}$, for energies greater than $13.6 \mathrm{eV}$. This slope is steeper than the generally observed one (typically $f_{v} \propto v^{-0.7}$ ) but is appropriate if the hard continuum strikes the cloud before encountering the Compton reflector. This is the geometry preferred, for instance, by Smith, Dane, \& Pounds (1993).

The relative luminosities of the soft and hard components, as seen by BLR clouds, is unknown, although it is known that the ratio of optical to X-ray flux, parameterized by the ultraviolet to $\mathrm{X}$-ray spectral index, $\alpha_{\mathrm{ox}}$, (Zamorani et al. 1981) is typically -1.4 . This is the spectral index which would describe the continuum between $2 \mathrm{keV}$ and $2500 \AA$ if the continuum could be described as a single power law, that is

$$
\frac{f_{v}(2 \mathrm{keV})}{f_{v}(2500 \AA)}=\left(\frac{v_{2 \mathrm{keV}}}{v_{2500 \AA}}\right)^{\alpha_{0 x}}=403.3^{\alpha_{0 x}} .
$$

This parameter is used to join the two spectral components, the $f_{v} \propto v^{0} \exp (-h v / k T)$ "big bump" or soft continuum, and the $f_{v} \propto v^{-1}$ hard continuum.

\subsection{Impact on Emission-Line Spectrum}

Figure 5 shows both the incident and transmitted continuum for our final model, discussed below. It is shown here to illustrate that the results are most sensitive to the continuum at unobservable energies. The gas we model absorbs the continuum at energies of typically $130 \mathrm{eV}$. We normalize the big bump and X-ray components through the ratio measured by $\alpha_{\mathrm{ox}}$. The dashed line on the figure connects these two points. The relevant continuum lies between these two measured points. Our assumed slope of the X-ray continuum also affects the results since high-ionization gas is most sensitive to the continuum near $100 \mathrm{eV}$. Thus, it is necessary to extrapolate the observed $2 \mathrm{keV}$ continuum point to the $100 \mathrm{eV}$ range, further increasing the uncertainty.

The family of AGNs are generally characterized by $\alpha_{o x}$ in the range -1 to -2 , although the net continuum striking the BLR could have a vastly different value if the UV continuum is either formed at larger radii than the BLR or if this continuum is formed in a flattened geometry such as a disk (Netzer 1987). Finally, $\alpha_{o x}$ may (Avni \& Tananbaum 1986) or may not (La Franca et al. 1995) depend on the continuum luminosity.

\subsection{Observational Constraints on the Continuum Shape}

The combination of the slope of the power law in equation one and the cutoff temperature were chosen to match the observed ultraviolet continuum of $0207-398$. The lack of curvature in the UV continuum of 0207-398 shows that the soft component must have a high-energy cutoff temperature of greater than $200,000 \mathrm{~K}$. Were it to have a temperaature much greater than $600,000 \mathrm{~K}$ it would extend further into the X-rays than the soft X-ray excess often seen in AGNs (Wilkes \& Elvis 1987). [These temperatures are appropriate for our choice of the optical continuum slope, $f_{v} \approx$ constant $\left.\times \exp \left(h v / k T_{\text {cutoff }}\right)\right]$.

The X-ray observations are confusing. Q0207-398 had an observed value of $\alpha_{o x}$ of -1.57 in 1982 (Wilkes et al. 1994) but -1.84 in 1992 (Bechtold et al. 1994), while the line spectrum did not undergo a major change. (We come back to this point below-the emission lines would have changed a lot had the ionizing continuum changed by the amount indicated by the change in $\alpha_{o x}$ ). Obviously $\alpha_{o x}$ is poorly known and may not be telling us much about the continuum striking the clouds if different continuum components are strongly beamed.

\subsection{Model Parameters}

In the following we will vary the continuum shape, both the high-energy cutoff temperature of the soft component and $\alpha_{\mathrm{ox}}$, to find the largest possible predicted $\mathrm{N} \mathrm{v} / \mathrm{He}$ II intensity ratio.

Given these assumptions concerning the shape of the continuum, the free parameters are the high-energy cutoff temperature for the big bump $T_{\text {cutoff }}$, the surface flux of ionizing photons [usually $\Phi(\mathrm{H})$, with units $\mathrm{cm}^{-2} \mathrm{~s}^{-1}$ ], the optical to X-ray spectral index $\alpha_{\mathrm{ox}}$, the cloud density $n_{\mathrm{H}}$, and the cloud column density $N_{\mathrm{H}}$. We assume constant density clouds to avoid the stability problems inherent in isobaric clouds at high radiation densities (Elitzur \& Ferland 1986; Mathews 1992). Theoretically, the pressure law describing the clouds is simply unknown. Magnetic support (Rees 1987) is a possibility, or the clouds may be the surface of a bound disk (Collin-Souffrin 1991). Constant density is the simplest assumption and the most useful in the exploratory work described here. The emitted spectrum is not sensitive to this assumption since here we consider only highly ionized gas, with nearly constant temperature. The clouds considered here reprocess mainly the continuum between 4 ryd and 50 ryd (see Fig. 5). Were we to use the traditional photon flux (the flux of hydrogen ionizing photons) changes in the continuum shape at constant flux would result in major changes in the flux of photons producing $\mathrm{C}$ IV, $\mathrm{N} \mathrm{v}$ and $\mathrm{He}$ II. The resulting emission-line spectrum would have changed because of changes in the effective ionization parameter, not because of real changes in the continuum. In the remainder of this paper, the flux we will consider will be the flux in $\mathrm{He}^{+1}$-ionizing radiation $\Phi\left(\mathrm{He}^{+}\right)$- the flux density of photons greater than 4 ryd. This parameterization better preserves the homology relations offered by the ionization parameter.

The observed kinematics show that the $\mathrm{N}$ v-He II emitting clouds must be matter-bounded (actually, optically thin in the $\mathrm{He}^{+}$continuum). Were the clouds to have a large column density a $\mathrm{N}^{+4}-\mathrm{N}^{+3}$ ionization front would occur, and (unobserved) broad N Iv] 1486 (along with many other lower ionization lines) would become strong. In our calculations the cloud column density is set by the requirement that the high-ionization region does not overemit unobserved low-ionization lines (see Table 3). The actual limits, expressed as intensities relative to $\mathrm{N}$ v 1240, were: $\mathrm{Si}$ IV $1397+\mathrm{O}$ IV] $1402<0.23 ; \mathrm{N}$ IV] $1486<0.06 ; \mathrm{O}$ III] $1666<0.09 ; \mathrm{Si} \mathrm{III]} 1895<0.02$; and C III] $1909<0.14$, N III $990<0.22$, C III $977<0.22$. In practice the N IV] 1486 limit usually stopped the models. As a result of our observationally based stopping criteria, the column density of each model is itself model-dependent, but is generally in the neighborhood of $10^{20}-10^{21} \mathrm{~cm}^{-2}$.

Finally, calculations are performed with version 87 of the radiative equilibrium code Cloudy, last described by Ferland (1995).

\section{THE FAILURE OF SOLAR ABUNDANCES}

This section describes our attempts to reproduce the observed N v 1240/He II 1640 intensity ratio using solar abundances (Grevesse \& Anders 1989). Our approach will 
be to search for parameters giving the largest possible $\mathrm{N} \mathrm{v/He} \mathrm{II} \mathrm{ratio,} \mathrm{which} \mathrm{for} \mathrm{solar} \mathrm{abundances} \mathrm{will} \mathrm{turn} \mathrm{out} \mathrm{to}$ nearly an order of magnitude smaller than observed. We tried both photoionization simulations using a variety of continuum shapes, hydrogen densities, column densities, and ionizing photon fluxes, and also collisional equilibrium models which invoke nonradiative heating with a range of resulting temperatures. None succeeded, leading to the conclusion that the nitrogen abundance has to be significantly enhanced in luminous quasars.

A major emphasis of this work will be to understand the large $\mathrm{N} \mathrm{V} \lambda 1240 / \mathrm{He}$ II $\lambda 1640$ ratio in component B of Q0207-398. Despite the advantage of being able to study kinematically distinct components in this object, the geometry and structure of the emitting region(s) producing the observed broad lines is still not well defined. Given this uncertainty, it is necessary to develop especially robust abundance indicators if the results are to be robust. We will show that the $\mathrm{N} \mathrm{v} / \mathrm{He}$ II intensity ratio for solar abundances is roughly 1 for a very wide range of continua shapes, intensities, and cloud densities, and has little dependence on cloud column density. The ratio also has little dependence on the uncertain geometry because the lines are formed in coextensive regions.

\subsection{The Density and Flux Grid}

The shape of the $\mathrm{N}$ v-producing continuum is unknown and unmeasurable. In the initial discussion we will adopt a fiducial continuum with an $\alpha_{o x}$ of -1.4 and a big bump cutoff temperature of $250,000 \mathrm{~K}$. Because of our choice of helium-ionizing flux as an independent parameter, where each model continuum produces the same flux of heliumionizing photons, the continuum shape will not greatly affect our results.

Figure 6 shows the results of a series of calculations in which the continuum shape was held fixed at $\alpha_{o x}=-1.4$ and $T_{\text {cutoff }}=250,000 \mathrm{~K}$, and the hydrogen density and flux of helium-ionizing photons were then varied. The figure shows a ridge running diagonally across the diagram, corre-

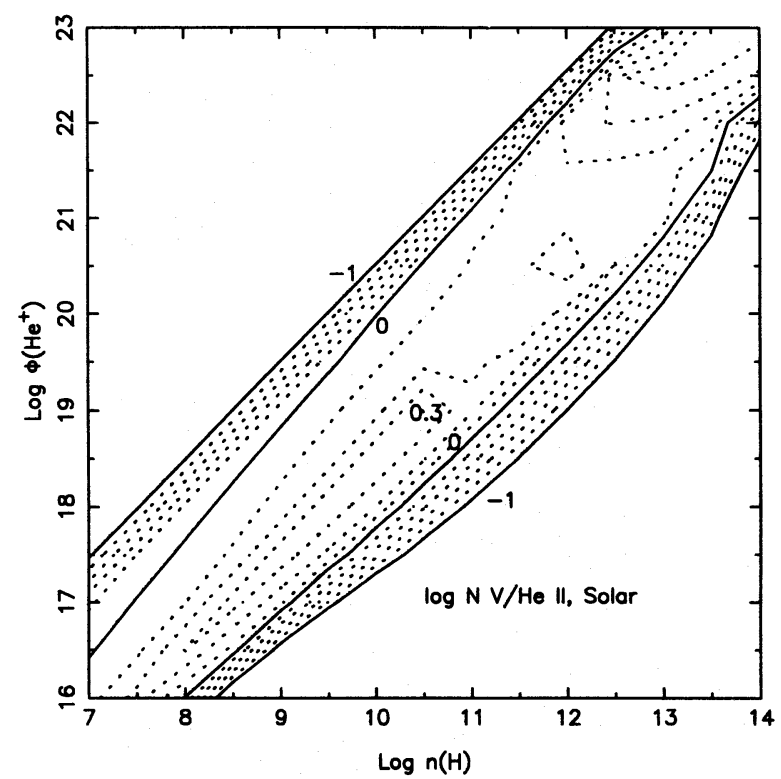

Fig. 6.-The $\mathrm{N} \mathrm{v/He} \mathrm{II} \mathrm{ratio} \mathrm{is} \mathrm{shown} \mathrm{as} \mathrm{a} \mathrm{function} \mathrm{of} \mathrm{the} \mathrm{density} \mathrm{and}$ flux in ionizing photons. The continuum had an $\alpha_{o x}$ of -1.4 and a big bump cutoff corresponding to a temperature of $250,000 \mathrm{~K}$. sponding to a constant ionization parameter. For this continuum shape and the adopted flux of helium-ionzing photons, the commonly quoted ionization parameter (the ratio of hydrogen-ionizing photon to hydrogen densities) is $U \equiv \Phi(\mathrm{H}) / n c \approx \frac{1}{2}$. This corresponds to conditions in which $\mathrm{N}^{+4}$ most nearly fills the $\mathrm{He}^{+2}$ zone. The peak line ratio along this ridge is roughly 2 at most densities, increasing to $\approx 5$ at the highest densities $\left(n \approx 10^{14} \mathrm{~cm}^{-3}\right)$ (Densities above $5 \times 10^{11} \mathrm{~cm}^{-3}$ are ruled out by the equivalent width of $\mathrm{N} \mathrm{v}$, shown in Fig. 7 discussed below). The ridge exists for two reasons. For low-ionization parameters $U$ (i.e., points below or to the right of the ridge) little $\mathrm{N}^{+4}$ is present and the gas is cool, so the $\mathrm{N} \mathrm{v/He} \mathrm{II} \mathrm{ratio} \mathrm{is} \mathrm{small.} \mathrm{For} \mathrm{large} U$ (the upper left corner of the figure) the gas equilibrium goes from the nebular phase $\left(T \approx 10^{4} \mathrm{~K}\right)$ to the Compton temperature ( $T_{\text {Compton }} \approx 6 \times 10^{6} \mathrm{~K}$ for this radiation field) and nitrogen is more ionized than $\mathrm{N}^{+4}$.

For reference, the well-studied Seyfert galaxy NGC 5548, for which line-continuum variability measurements have been made (Clavel et al. 1991; Krolik et al. 1991), has a flux of helium-ionizing photons in the neighborhood of $10^{19.8}$ $\mathrm{cm}^{-2} \mathrm{~s}^{-1}$ (for a distance of 10 light days, and the observed continuum shape for NGC 5548) and a density of roughly $10^{11} \mathrm{~cm}^{-3}$ (Ferland et al. 1992). NGC 5548 has $\mathrm{N} \mathrm{v/}$ $\mathrm{He}$ II $\sim 1$ consistent with solar abundances.

Figure 7 shows the results of a series of calculations in which the ionization parameter was held constant at a value appropriate to the peak of the ridge, and the density varied. Specifically the ratio $\Phi\left(\mathrm{He}^{+}\right) / n$ was kept constant at the value $10^{9} \mathrm{~cm} \mathrm{~s}^{-1}$. The equivalent width of $\mathrm{N} \mathrm{v} \lambda 1240$, and

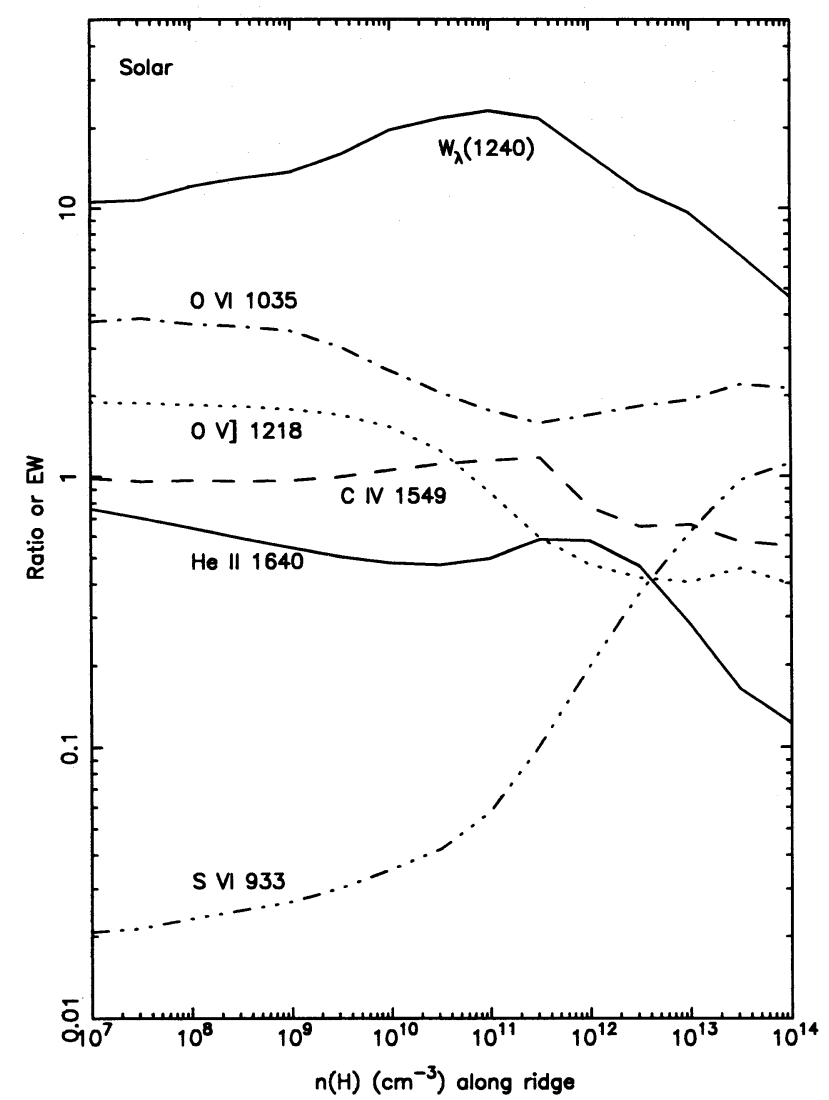

FIG. 7.-The intensities of several emission lines relative to $N$ v 1240 , and the equivalent width of $\lambda 1240$, are shown as a function of the density and flux in ionizing photons for pairs chosen to lie along the ridge in the previous figure. Equivalent widths are for full coverage. 
the intensities of several of the stronger lines relative to this line, are shown. The equivalent width of $\mathrm{N} v$ is computed assuming that clouds fully cover the continuum source. It initially increases because the gas grows hotter as other coolants, with lower critical densities, are suppressed, and the cooling shifts over to $\mathrm{N}$. Eventually, for $n>10^{11}$ $\mathrm{cm}^{-3} \mathrm{~N} \mathrm{~V}$ too is suppressed and the equivalent width decreases with increasing density. The largest equivalent width ever achieved, $\approx 25 \AA$ for full coverage, is 2.5 times that assigned to the component B observed in Q0207-398. The limit to the intensity of S vi 933 (Table 3) sets the upper limit to the density of $n \leq 10^{12} \mathrm{~cm}^{-3}$. For densities $\approx 10^{11}$ $\mathrm{cm}^{-3}$, where the equivalent width is largest, the predicted $\mathrm{N}$ v/O vi ratio is roughly 6 times smaller than observed, and predicted $\mathrm{N} v / \mathrm{He}$ II ratio is $\approx 6$ times smaller than the observed limit.

\subsection{The Continuum Shape Grid}

Figure 8 shows the results of calculations in which the continuum parameters $T_{\text {cutoff }}$ and $\alpha_{\text {ox }}$ were varied as described above. The cloud density was held constant at $n_{\mathrm{H}}=10^{11} \mathrm{~cm}^{-3}$, the flux of helium ionizing photons held constant at $\Phi\left(\mathrm{He}^{+}\right)=10^{20} \mathrm{~cm}^{-2} \mathrm{~s}^{-1}$, and solar abundances (Grevesse \& Anders 1989) were used. This flux and density were chosen to produce the largest possible $\mathrm{N} v$ / He II ratio and equivalent width of $\mathrm{N} v$, as described above.

The $\mathrm{N} \mathrm{V} / \mathrm{He}$ II ratios are the result of the interplay between the hard power-law and thermal components, the two components illustrated in Figure 5. For soft $\alpha_{o x}$ (closer to -2 ), the bump dominates the ionizing continuum and the results depend mostly on the bump temperature. For harder $\alpha_{o x}$ there is an interplay between the bump and power-law contributions. The ionization potential of $\mathrm{He}^{+2}$ is $54 \mathrm{eV}$, while the ionization potential of $\mathrm{N}^{+4}$ is higher, 77 eV. The $\mathrm{N}^{+4}$ region most nearly filled the $\mathrm{He}^{+2}$ zone when the incident continuum over the $50-80 \mathrm{eV}$ region is very flat in $v f_{v}$. For higher bump temperatures the $\mathrm{N} v$-producing portion of the bump is on the Wien tail of its energy dis-

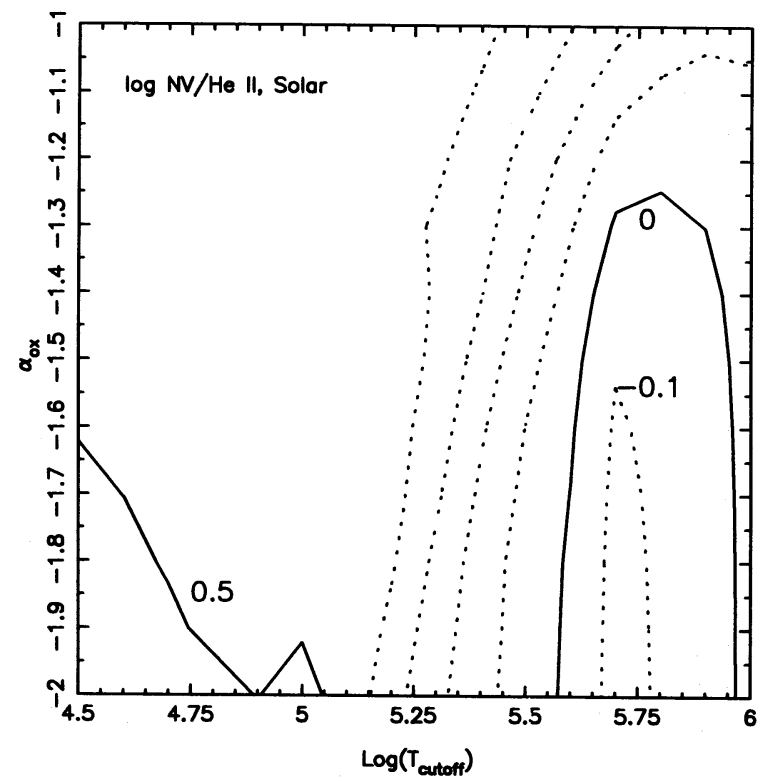

FIG. 8.-The $\mathrm{N} v / \mathrm{He}$ II ratio is shown as a function of the cutoff temperature of the big bump, and the ratio of optical to X-ray continua flux densities, $\alpha_{o x}$. The constant hydrogen density was $10^{11} \mathrm{~cm}^{-3}$, and the flux of helium-ionizing photons was $10^{20} \mathrm{~cm}^{-2} \mathrm{~s}^{-1}$. tribution. This selectively creates $\mathrm{He}^{+2}$ with little $\mathrm{N}^{+4}$ because of the exponential decline between the two ionization potentials. The result is that the highest $\mathrm{N} \mathrm{v/He}$ II ratio with significant equivalent width is produced by continuum shapes where the X-ray power law dominates above $54 \mathrm{eV}$. This occurs when the bump is coolest, or $\alpha_{o x}$ largest, as in the upper left corner of the figure. This ratio is about 2 . Even larger ratios can be reached in the small $\alpha_{o x}-$ lowtemperature bump corner, but this is due to continuum fluorescent production of the lines, the equivalent width of $\mathrm{N} \mathrm{v}$ is very small, and the continuum does not match the observations.

To summarize: the $\mathrm{N} v$ line is too weak for solar abundances, even with the "best case" density, flux, and continuum shape. We next discuss whether this could be an artifact of an incorrect assumed geometry.

\subsection{Dependence on Geometry or Column Density}

The general problem of the too-strong $\mathrm{N} \mathrm{v}$ emission, relative to such lines as $\mathrm{Ly} \alpha$ or $\mathrm{C}$ IV, is an old one and has been well documented (Baldwin \& Netzer 1978; Davidson \& Netzer 1979). One suggested explanation was that the $\mathrm{N} \mathrm{v}$ emission originated in a region of small column density, which produced $\mathrm{N} v$ but little $\mathrm{C}$ IV or Ly $\alpha$. This is possible since the ionization potential of $\mathrm{N}^{+4}$ is higher than that of $\mathrm{C}^{+3}$ or $\mathrm{H}^{+}$, so that these lines originate in different regions even within the same cloud. We show here that, although this geometry could explain the strength of $\mathrm{N} v$ relative to $\mathrm{C}$ IV or Ly $\alpha$, it does nothing to explain the $\mathrm{N} \mathrm{v} / \mathrm{He}$ II ratio. This is because any region producing $\mathrm{N} v$ must also make He II.

The upper panel of Figure 9 shows the depth-weighted emissivity of several of the stronger BLR lines as a function of depth into a cloud. The quantity plotted is the emergent intensity of the line per unit volume $\left(h v n_{u} A_{u, l} P_{u, l}\right.$, where $n_{u}$ is the density in the upper level of the transition, $A_{u l}$ is the transition probability, and $P_{u l}$ is the escape probability) multiplied by the depth into the cloud $D$. Dimensionally, this quantity is similar to the emergent line intensity, and on a log-log plot (which tends to emphasize regions with small depth or intensity) weights the plotted quantity to properly reflect contributions to the total observed intensity coming from each depth. The upper panel is for solar abundances, the standard continuum assumed above, a flux of heliumionizing photons of $10^{20} \mathrm{~cm}^{-2} \mathrm{~s}^{-1}$, and a density of $10^{11}$ $\mathrm{cm}^{-3}$. The lower panel is for enhanced abundances, and is described later.

The $\mathrm{N} \mathrm{v/He} \mathrm{II} \mathrm{ratio} \mathrm{sets} \mathrm{a} \mathrm{reliable} \mathrm{lower} \mathrm{limit} \mathrm{to} \mathrm{the} \mathrm{N} / \mathrm{He}$ abundance ratio because the ionization potentials constrain the lines to be formed in nearly identical regions. Although is possible to contrive geometries in which Ly $\alpha$ and $C$ IV are not formed in the same region as $\mathrm{N} \mathrm{v}, \mathrm{He}$ II must be because of the ionization potentials. If only a portion of the geometry shown in Figure 9 is actually present, then the $\mathrm{N} \mathrm{v}$ and $\mathrm{He}$ II emission will still track one another.

The line profile observations described in $\S 2$ suggest that Ly $\alpha$ and $\mathrm{N} v$ are not formed in the same region in Q0207-398. Figure 9 shows that this is a natural consequence of a BLR geometry in which the clouds forming the high-ionization lines are fully ionized (do not extend to the $\mathrm{H}^{+}-\mathrm{H}^{0}$ ionization front).

We conclude that the too-large $\mathrm{N} v / \mathrm{He}$ II ratio cannot be produced with solar abundance by altering the assumed geometry, i.e., by adding a component of fully ionized gas. 


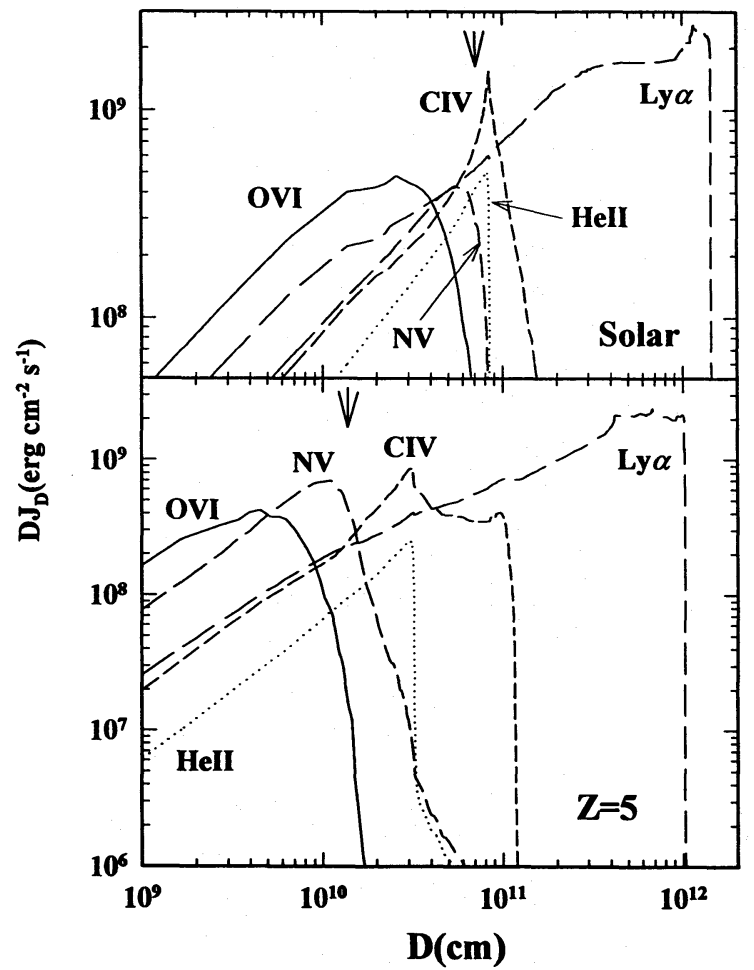

Fig. 9.-This shows the depth-weighted emissivity of several lines for solar abundances (upper panel) and for a metallicity 5 times solar (lower panel). The calculations are for a flux of helium-ionizing photons of $10^{20}$ $\mathrm{cm}^{-2} \mathrm{~s}^{-1}$ and a density of $10^{11} \mathrm{~cm}^{-3}$. The $\mathrm{N} \mathrm{v}$ and $\mathrm{He}$ II emitting regions are nearly cospatial, while $\mathrm{C}$ IV and $\mathrm{Ly} \alpha$ are formed at increasingly greater depths into the cloud. The arrows point to the depth where the calculation would have stopped, given our limits to the intensities of lower ionization lines.

\subsection{Required $\mathrm{N}$ v-Region Temperature}

Our basic problem is to match the observed $\mathrm{N} v / \mathrm{He}$ II ratio with solar abundances. The photionization simulations presented above fail, basically because the gas is too cool. $\mathrm{N} \mathrm{v}$ is collisionally excited so its intensity has an exponential temperature dependence. He II is a recombination line and decreases roughly linearly with temperature. What temperature is needed to reproduce the observed limit with solar abundances? A simple estimate of the lowest temperature needed to reach the observed lower limits can be made by assuming that the $\mathrm{N} \mathrm{v}$ line is optically thin and that the $\mathrm{He}$ II line emits under case B conditions. Using He II emissivities from Hummer \& Storey (1987) for a density of $10^{10} \mathrm{~cm}^{-3}$, and the $\mathrm{N} \mathrm{v}$ collision strength quoted by Mendoza (1983), we can set the ratio

$$
\frac{I(1240)}{I(1640)}=\frac{1640}{1240} \frac{\mathrm{N}^{+4}}{\mathrm{He}^{+2}} \frac{q_{l, u}(1240)}{\alpha_{\mathrm{eff}}(1640)},
$$

where $q_{l, u}$ is the rate coefficient for electron collisional excitation and $\alpha_{\text {eff }}$ is the effective recombination coefficient for 21640. Approximating $\alpha_{\text {eff }}$ as a power law in the temperature and assuming a solar $\mathrm{N} / \mathrm{He}$ ratio we find

$$
\frac{I(1240)}{I(1640)}=170\left[\frac{\mathrm{N}^{+4} / \mathrm{N}}{\mathrm{He}^{+2} / \mathrm{He}}\right] t_{4}^{0.48} \exp \left(\frac{-116.1}{t_{4}}\right),
$$

where $t_{4} \equiv T / 10^{4} \mathrm{~K}$. The term in brackets is the ionization correction factor (ICF), the ratio of the volume of the $\mathrm{N}^{+4}$ zone relative to that of the $\mathrm{He}^{+2}$ zone. This ratio is always less than one because of the ionization potentials $-\mathrm{He}^{+2}$ always dominates in any high ionization region, while Li-like $\mathrm{N}^{+4}$ is not favored $\left(\mathrm{N}^{+5}\right.$, which has a closed shell, is far more robust). The ICF is typically in the range $0.1-0.5$ for the models presented above, and we take the higher number to make the more conservative case. Solving equation (4) for a ratio of 10 , and an ICF of 0.5 , we see that a temperature of $\sim 41,000 \mathrm{~K}$ is needed to reproduce the observed limit. The actual temperatures found in the $\mathrm{N}^{+4}$ zone of a BLR cloud are more like $20,000 \mathrm{~K}$, and temperatures as high as $40,000 \mathrm{~K}$ cannot be reached with photoelectric heating because cooling by collisionally excited lines is too efficient. For a gas in photoionization equilibrium the temperature is set by energy conservation. The real reason solar abundances cannot reproduce the observed ratio is because, with solar abundances and photoelectric heating, the cloud is able to cool efficiently via $\mathrm{O}$ VI and $\mathrm{C}$ IV lines, without making strong $\mathrm{N} \mathrm{v}$.

\subsection{Collisional Ionization with Solar Abundances}

Since we cannot find a photoionization model which will produce the observed $\mathrm{N} \mathrm{v} / \mathrm{He}$ II ratio with solar abundances, we now try a collisionally ionized model.

Figure 10 shows a series of calculations in which the radiation field is set to a very low intensity, the electron temperature varied between low and high values, and the hydrogen density was fixed at $10^{10} \mathrm{~cm}^{-3}$. The model is entirely collisionally ionized. Table 4 lists predictions of the models. The emission from a thin cell of gas is computed, and the ratios of some of the stronger observed lines are plotted in the figure. As expected, the $\mathrm{N} \mathrm{v/He} \mathrm{II} \mathrm{ratio} \mathrm{does}$ indeed get large when the temperature is large. This is because the intensity of a recombination line (like $\mathrm{He}$ II)

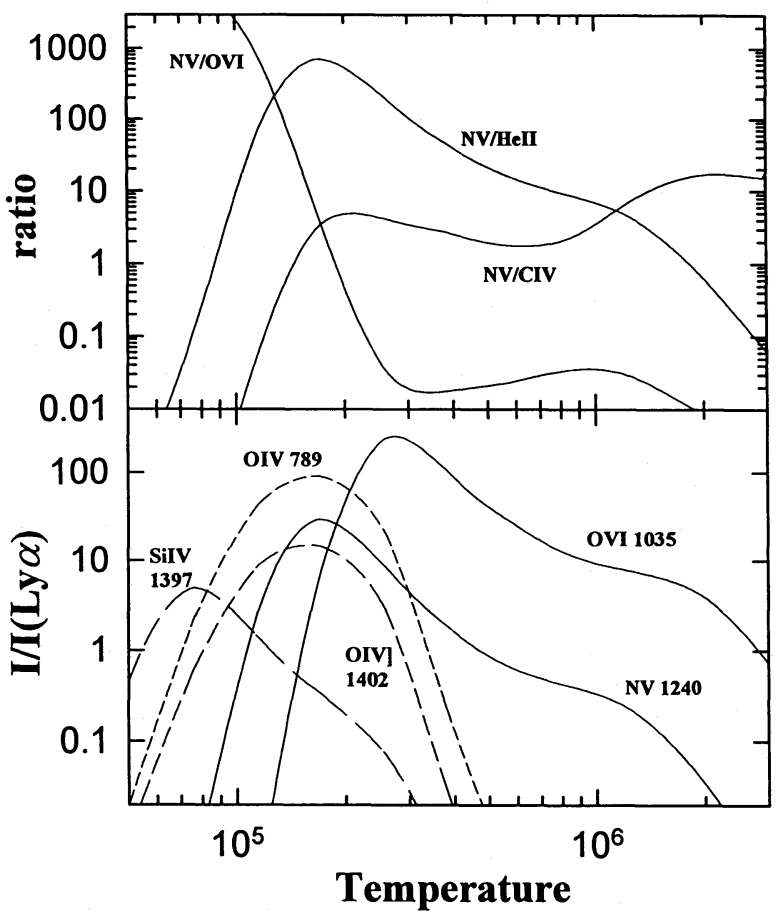

Fig. 10.- The intensities of the strongest lines produced in collisional equilibrium at solar abundances at a density of $10^{10} \mathrm{~cm}^{-3}$. The simulation is of an optically thin cell of gas under conditions of collisional ionization equilibrium. Temperatures in the range $10^{5} \mathrm{~K}$ to $10^{6} \mathrm{~K}$ are consistent with the observed limit to $\mathrm{N} v / \mathrm{He}$ II, while the observed $\mathrm{O} \mathrm{vI} / \mathrm{N} \mathrm{v}$ ratio sets the temperature to $189,000 \mathrm{~K}$. At this temperature $\mathrm{C}$ IV is predicted to be 4 times weaker than is observed. 
TABLE 4

Collisional Equilibrium ${ }^{a}$

\begin{tabular}{cccc}
\hline \multicolumn{1}{c}{ Line } & $100,000 \mathrm{~K}$ & $200,000 \mathrm{~K}$ & $400,000 \mathrm{~K}$ \\
\hline O v $630 \ldots \ldots \ldots$ & 0.974 & 10.37 & 4.57 \\
O IV $789 \ldots \ldots \ldots$ & 46.7 & 3.26 & 0.0858 \\
S vi $933 \ldots \ldots \ldots$. & 0.0145 & 0.154 & 0.234 \\
O vi $1035 \ldots \ldots$. & 0.0003 & 2.09 & 55.5 \\
H I $1216 \ldots \ldots \ldots$ & 2.06 & 0.0346 & 0.580 \\
O v] $1218 \ldots \ldots$. & 0.195 & 0.987 & 0.272 \\
N v $1240 \ldots \ldots$. & 1 & 1 & 1 \\
Si IV $1397 \ldots \ldots$. & 4.74 & 0.0092 & 0.0020 \\
O Iv] $1402 \ldots \ldots$. & 10.4 & 0.446 & 0.0108 \\
C IV $1549 \ldots \ldots$. & 122 & 0.214 & 0.384 \\
He II $1640 \ldots \ldots$. & 0.0729 & 0.0016 & 0.0216 \\
\hline
\end{tabular}

a Collisional equilibrium models with solar abundances and a density of $10^{10} \mathrm{~cm}^{-3}$

goes down with increasing temperature, while that of a collisionally excited line $(\mathrm{N}$ v) goes up until that ion is itself collisionally ionized away. As can be seen from Figure 10 temperatures in the range between $10^{5} \mathrm{~K}$ and $10^{6} \mathrm{~K}$ reproduce the observed limit to the $\mathrm{N} \mathrm{v/He}$ II ratio.

The observed $\mathrm{N} v / \mathrm{O}$ vI intensity ratio measures the temperature. The observed ratio is met only near $189,000 \mathrm{~K}$. The $\mathrm{N} \mathrm{v/C} \mathrm{IV} \mathrm{ratio} \mathrm{is} \approx 4$ times too large here, however.

Table 4 gives a more complete list of the predictions for three temperatures spanning the range of possibilities. Although collisional ionization can account for the $\mathrm{N} \mathrm{v} /$ $\mathrm{He}$ II ratio, it violates several of the other line ratios. A last problem with this scenario is that gas in this temperature range is thermally unstable (Dalgarno \& McCray 1973), and so would tend to run away to coronal temperatures on a thermal timescale (of order $10 \mathrm{~s}$ for the $2 \times 10^{5} \mathrm{~K}$ model, with a density of $10^{10} \mathrm{~cm}^{-3}$ ). We also computed denser models $\left(10^{11} \mathrm{~cm}^{-3}\right)$, and models with column densities $10^{20}-10^{21} \mathrm{~cm}^{-2}$. The conclusions reached here were not changed.

Collisional ionization does not help and is not in keeping with evidence from reverberation studies that the gas is photoionized. It will not be discussed further.

\section{ENHANCED ABUNDANCES}

Section 4 showed that solar abundances are not able to reproduce the $\mathrm{N} v$ intensity relative to other lines in $0207-398$. What is needed is a way to selectively enhance the strength of N v. As was described in the Introduction, high metallicities accompanied by enhanced $\mathrm{N}$ should have this effect. Here we investigate that possibility.

We can estimate the magnitude of the required overabundance using the arguments in $\S$ 4.4. For a temperature of $20,000 \mathrm{~K}$ (typical of the high-ionization zone of a BLR model) the predicted $\mathrm{N} \mathrm{V} / \mathrm{He}$ II is 0.36 , roughly 28 times smaller than our limit of 10 . Assuming that nitrogen, a secondary element, goes up as the square of the metallicity, we anticipate that a metallicity of 5 times solar will be needed to match the $\mathrm{N} \mathrm{v/He} \mathrm{II} \mathrm{ratio.}$

\subsection{Abundances from Stellar Evolution}

We raise the global metallicity, and the specific abundances of the elements, following the calculations of Hamann \& Ferland (1993). These calculations are guided by observations of enriched metallicities in the cores of massive galaxies (Arimoto \& Yoshii 1986; Bica 1988; Bica et al. 1988). The free parameters are the slope of the initial mass function, a possible low-mass limit to the IMF, and the rate at which primordial gas forms stars. Given these parameters, the mix of abundances is fully prescribed, with only one free parameter, the age of the cluster, or equivalently, the global metallicity $Z$.

The evolution models are described fully by Hamann \& Ferland (1993). We use an evolution model (M5a of that paper), where the star formation and infall timescales are very close to, but slightly faster than, the "standard" elliptical model (see also Arimoto \& Yoshii 1987; Matteucci \& Greggio 1986; Matteucci \& Tornambe 1987; Bica 1988). Its IMF also has a slope very similar to, but slightly steeper than, that of the standard elliptical model $(x=1.0$ instead of 1.1). The main difference is that the IMF has a lower mass cutoff at $M=2.5 M_{\odot}$ instead of $\sim 0.1 M_{\odot}$ in the standard models. The truncated IMF that distinguishes the abundance set we use from the standard elliptical model is similar to that estimated for starburst galaxies with no lowmass stars forming (Scalo 1990). It allows the gas to reach mugh higher metallicities (up to $35 Z_{\odot}$ ) before becoming locked up in stellar remnants. (Low-mass stars can tie up a great deal of gas while adding little to the global enrichment of the system.)

We adopt the truncated IMF scenario here because it gives us the flexibility to examine very large metallicities. We note, however, that for metallicities $\$ 10 Z_{\odot}$, the abundance mixture is essentially the same as in the more standard evolution scenarios (see HF93). The lowest (and most conservative) metallicity which can accommodate our $\mathrm{N} \mathrm{v}$ intensity will turn out to be in the neighborhood of $5 Z_{\odot}$. In much of the following we will consider models with this metallicity in great detail. The specific abundances used for this minimum $5 Z_{\odot}$ case are, by number, $\mathrm{H}: 1.00$; $\mathrm{He}: 1.24 \times 10^{-1} ; \quad \mathrm{Li}: 1.19 \times 10^{-8} ; \quad \mathrm{Be}: 1.52 \times 10^{-10}$; B: $4.45 \times 10^{-9} ; \quad$ C: $8.24 \times 10^{-4} ; \quad \mathrm{N}: 1.15 \times 10^{-3}$; O: $5.95 \times 10^{-3} ; \quad \mathrm{F}: 1.95 \times 10^{-7} ; \quad \mathrm{Ne}: 7.94 \times 10^{-4}$; $\mathrm{Na}: 1.36 \times 10^{-5} ; \quad \mathrm{Mg}: 2.51 \times 10^{-4} ; \quad \mathrm{Al}: 1.93 \times 10^{-5} ;$ Si: $2.29 \times 10^{-4} ; \quad \mathrm{P}: 2.41 \times 10^{-6} ; \quad$ S: $1.06 \times 10^{-4}$; $\mathrm{Cl}: 1.21 \times 10^{-6} ; \quad \mathrm{Ar}: 2.34 \times 10^{-5} ; \quad \mathrm{K}: 8.72 \times 10^{-7} ;$ Ca: $1.42 \times 10^{-5} ; \quad$ Sc: $1.73 \times 10^{-9} ; \quad$ Ti: $1.22 \times 10^{-7}$; $\mathrm{V}: 1.49 \times 10^{-8} ; \quad \mathrm{Cr}: 6.87 \times 10^{-7} ; \quad \mathrm{Mn}: 4.85 \times 10^{-7} ;$ Fe: $6.64 \times 10^{-5} ; \quad$ Co: $3.18 \times 10^{-9} ; \quad \mathrm{Ni}: 2.50 \times 10^{-6}$; $\mathrm{Cu}: 2.65 \times 10^{-8} ; \mathrm{Zn}: 6.41 \times 10^{-8}$. In the following we shall refer to this as a metallicity of $5 Z_{\odot}$. Note that this mixture has a nitrogen abundance intermediate between those of carbon and oxygen and the N/C and N/He ratios are 4.5 and 8.1 times solar, respectively.

\subsection{The Density, Flux, and Continuum Grids at $Z=5 Z_{\odot}$ 5.2.1. The $\mathrm{N} \mathrm{v} / \mathrm{He}$ II Ratio vs. Flux and Density Grid}

Figure 11 shows the computed $\mathrm{N}$ v/He II ratio as a function of the flux of ionizing photons and density. This is computed for $Z=5 Z_{\odot}, \alpha_{o x}=-1.4$, and $T_{\text {cutoff }}=250,000$ $\mathrm{K}$. The figure shows a ridge running along a locus of density and flux corresponding to an ionization parameter of roughly $\frac{1}{2}$, as was the case for solar abundances (see the discussion in $\S 4.1$. above). The ridge is present for reasons very similar to those described in that section.

Figure 12 shows the $\mathrm{N} v$ equivalent width and intensities of several emission lines relative to $\mathrm{N} \mathrm{v}$ for positions along the ridge. The equivalent width decreases at the highdensity flux end of the ridge, largely because the line saturates near the blackbody limit. Given the need to have a substantial fraction of the $\mathrm{N} \mathrm{v}$-making continuum remain 


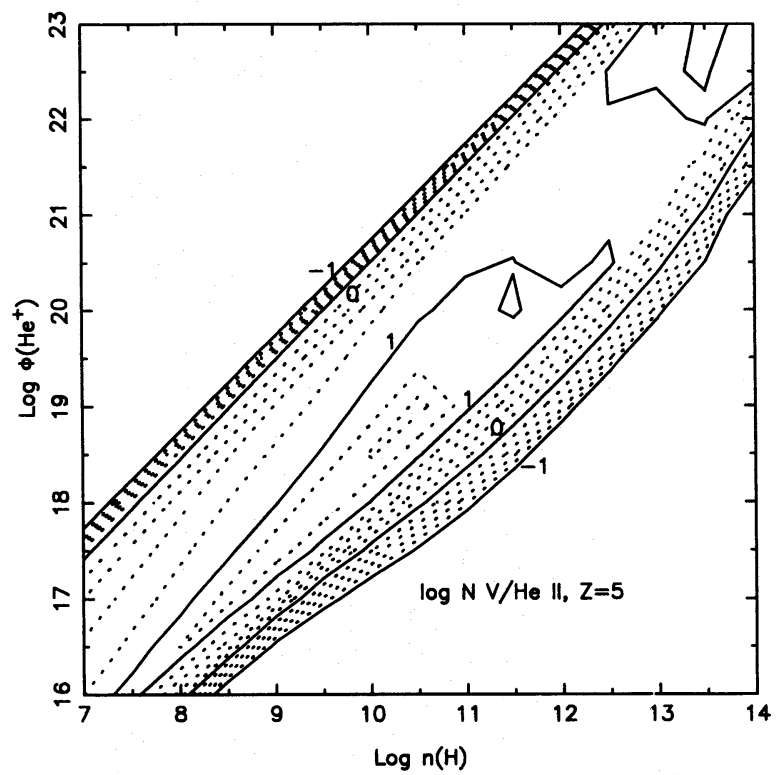

Fig. 11. - The $\mathrm{N} v / \mathrm{He}$ II vs. flux and density grid for $Z=5 Z_{\odot}$. This grid is for a value of $\alpha_{\mathrm{ox}}$ of -1.4 and a big bump cutoff corresponding to a temperature of $250,000 \mathrm{~K}$.

unabsorbed by component $\mathrm{B}$ (to produce the component $\mathrm{C}$ $\mathrm{N} v$ discussed in Paper 1), we need covering factors of less than $\frac{1}{2}$, leading to a limit of densities between $5 \times 10^{9}$ $4 \times 10^{12} \mathrm{~cm}^{-3}$, but the absence of detectable S vi $\lambda 933$ sets a limit to the density of $10^{12} \mathrm{~cm}^{-3}$. Densities in the neighborhood of $10^{11} \mathrm{~cm}^{-3}$ seem most able to reproduce the

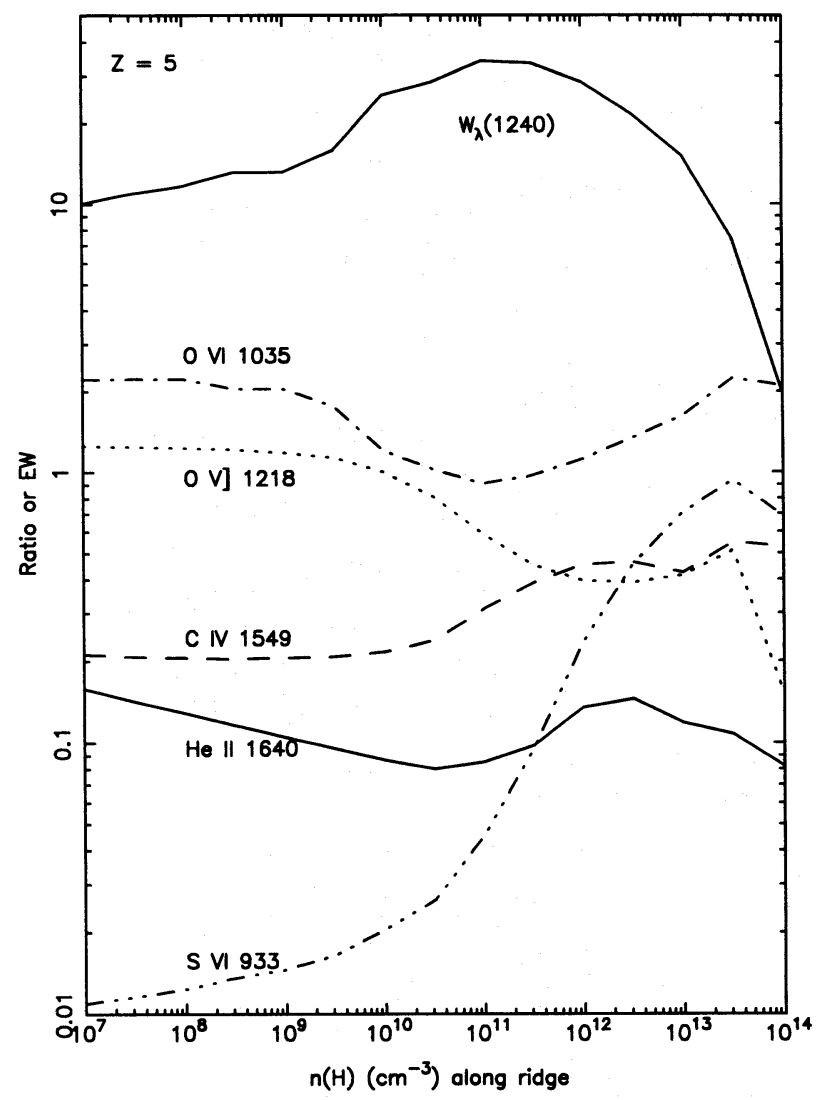

FIG. 12.-The emission-line spectrum as a function of position along the ridge. Line intensities are given relative to $\mathrm{N} v$. This grid is for $Z=5$ $Z_{\odot}$ and a ratio $\Phi\left(\mathrm{He}^{+}\right) / n=10^{9} \mathrm{~cm} \mathrm{~s}^{-1}$. Equivalent widths are for full coverage.
$\mathrm{N}$ v-He II spectrum. The $\mathrm{C}$ IV/ $\mathrm{N} v$ ratio at this point is roughly half the observed ratio. The final model presented below will use parameters slightly off the ridge and will reproduce this ratio.

\subsubsection{The $\mathrm{N} v / \mathrm{He}$ II ratio vs. Continuum Shape Grid}

Figure 13 shows the $\mathrm{N} v / \mathrm{He}$ II ratio as a function of the continuum shape. Both $\alpha_{o x}$ and the soft component cutoff temperature $T_{\text {cutoff }}$ are varied. The figure was computed with a density of $n=10^{11} \mathrm{~cm}^{-3}$ and a flux of ionizing photons of $\Phi\left(\mathrm{He}^{+}\right)=10^{20} \mathrm{~cm}^{-2} \mathrm{~s}^{-1}$. This density and flux were chosen to lie along the $\mathrm{N}$ v/He II ridge in Figure 11.

Figure 13 is very similar to Figure 8 (the continuum grid for solar abundances) in that it shows a general increase of $\mathrm{N} \mathrm{v/He}$ II in the direction of the upper left corner. But the values now range from 5 to 16 , just reaching beyond our most reasonable lower limit, given in column (2) of Table 3 , $\sim 9$.

\subsubsection{N v Equivalent Width vs. Continuum Shape Grid}

Figure 14 shows the equivalent width of $N \mathrm{v} \lambda 1240$ for various continuum shapes, a density of $10^{11} \mathrm{~cm}^{-3}$, a flux of helium-ionizing photons of $10^{20} \mathrm{~cm}^{-2} \mathrm{~s}^{-1}$, a metallicity of 5 $Z_{\odot}$, and full coverage of the continuum source by emitting gas. The equivalent width is much like the Zanstra ratio (Osterbrock 1989) in that it is sensitive to the number of high-energy photons relative to an observed (low-energy) continuum.

Our best estimate of the total equivalent width of $\mathrm{N} \mathrm{v}$ $\lambda 1240$ is $19.8 \AA$ (Paper I) and we assign $9.7 \AA$ of that to component B (Table 3). The big bump temperature must be less than $T \approx 600,000 \mathrm{~K}$; otherwise, it would extend into the X-rays. The observed UV continuum suggests that the big bump is hotter than $200,000 \mathrm{~K}$. Given these constraints, and the $\mathrm{N} \mathrm{v/He}$ II ratio (Fig. 13), we need values of $\alpha_{o x}>$ -1.45 to get a large enough $\mathrm{N} v$ equivalent width with a covering factor less than $\frac{1}{2}$. We come back to the issue of the $\mathrm{X}$-ray continuum below (\$ 5.3).

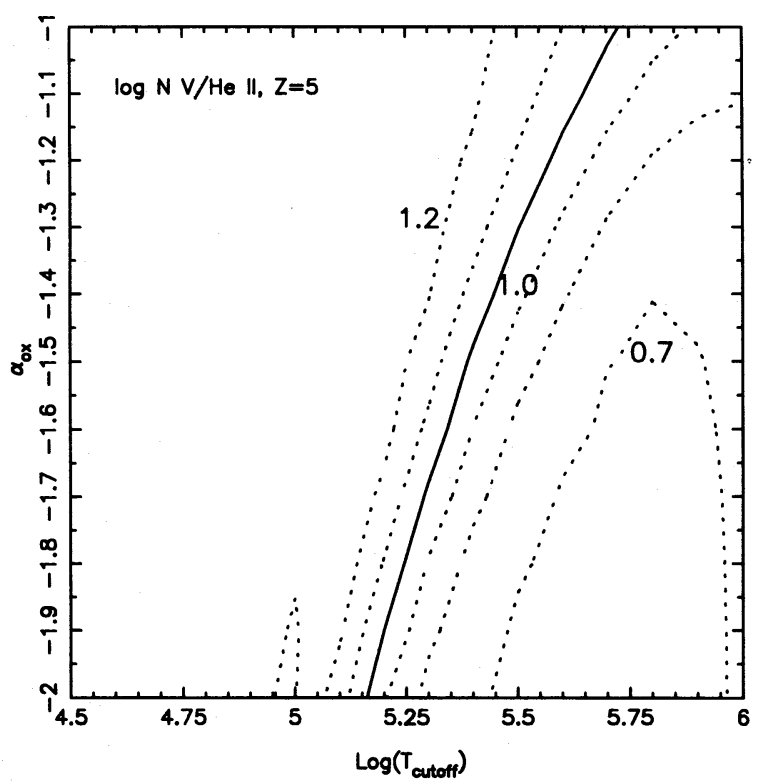

FIG. 13.-The $\mathrm{N}$ v/He II vs. continuum shape grid for a metallicity of 5 times solar. The continuum shape is varied with the density and flux of helium-ionizing photons left at values of $10^{11} \mathrm{~cm}^{-3}$ and $10^{20} \mathrm{~cm}^{-2} \mathrm{~s}^{-1}$, respectively. 


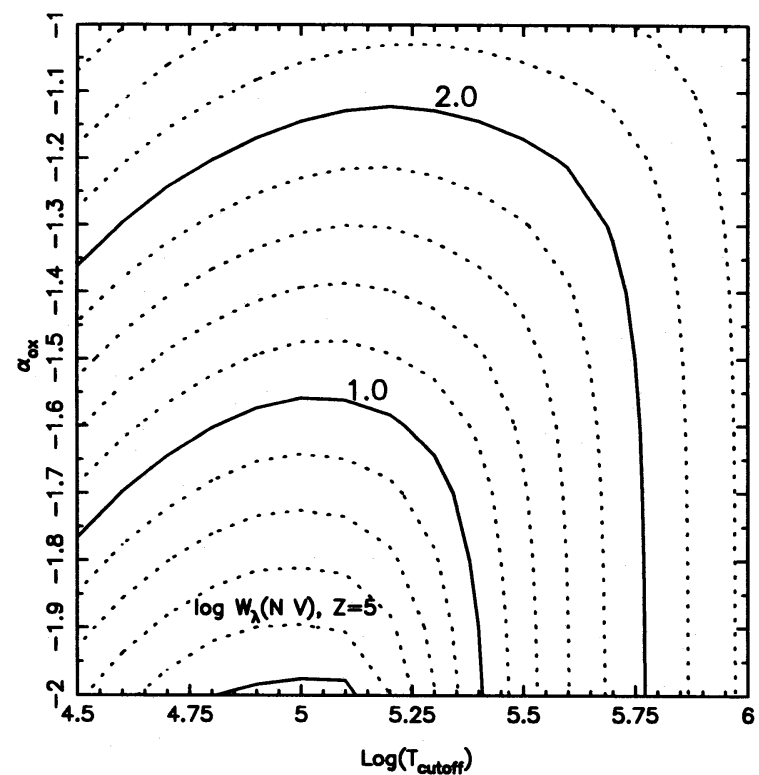

Fig. 14. - The $\mathrm{N} v$ equivalent width $\mathrm{vs.} \mathrm{continuum} \mathrm{shape} \mathrm{grid} \mathrm{for} \mathrm{a}$ metallicity of 5 times solar. The continuum shape is varied with the density and flux of helium-ionizing photons left at values of $10^{11} \mathrm{~cm}^{-3}$ and $10^{20}$ $\mathrm{cm}^{-2} \mathrm{~s}^{-1}$, respectively.

\subsubsection{The Structure of Clouds wuth High Metallicity}

This discussion has shown that a metallicity of $5 Z_{\odot}$ is just able to reproduce both the limit to the $\mathrm{N} v / \mathrm{He}$ II ratio and the observed equivalent width of $\mathrm{N} \mathrm{v}$ in Q0207-398. We adopt this metallicity to describe the high ionization region in the following discussion.

The lower panel of Figure 9 shows the emissivity structure of a BLR cloud for this metallicity. Except for the differences in metallicities, the lower and upper panels are computed for identical cloud density and flux of ionizing photons. Two striking differences are apparent. The first is that $N v \lambda 1240$ is much stronger relative to the other lines at the higher metallicity. This is because the nitrogen abundance is selectively enhanced relative to the other heavy elements, and so $\mathrm{N} \mathrm{v}$ carries a disproportionate share of the total cooling.

The second difference is that the scale of the entire structure is more compressed for the high-metallicity case. This is caused by the enhanced heavy-element opacities. Although hydrogen is still the most common element in our $5 Z_{\odot}$ mix, the abundant heavy elements absorb a large majority of the high-energy radiation and the incident flux is attenuated over a smaller depth.

\subsubsection{The Effects of Varying the Metallicity}

The effects of enhancing the heavy elements are further illustrated in Figure 15. The equivalent width of five of the lines we have concentrated on are shown as a function of the metallicity relative to solar. In these calculations the continuum shape and flux of ionizing photons was held fixed, while the metallicity was increased to its asymptotic limit $(\approx 35$ for this IMF). The electron density rather than the hydrogen density was held fixed as $Z$ was varied. This was necessary because at the higher metallicities $(Z \gtrsim 10$ $Z_{\odot}$ ) the heavy elements become increasingly important sources of free electrons. Were the hydrogen density to have been kept constant the free electron density would have increased with $Z$, and the gas would have become more

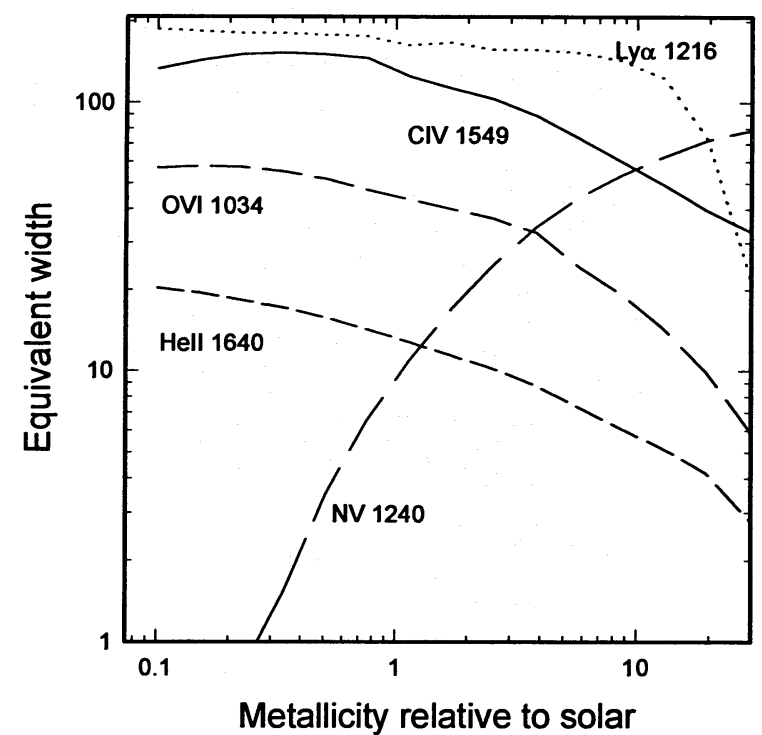

Fig. 15.-The equivalent width of He II $\lambda 1640, \mathrm{C}$ IV $\lambda 1549, \mathrm{O}$ vi $\lambda 21034$, and $\mathrm{N} v \lambda 1240$, as a function of the metallicity. All equivalent widths are referenced to the incident continuum at $1215 \AA$. The equivalent width of $\lambda 1640$ decreases with $Z$ as the heavy elements contribute an ever increasing fraction of the total gas opacity, and absorb more of the helium-ionizing radiation.

neutral as a result. By keeping the electron density constant we also keep the mean level of ionization constant, and so are able to better compare similar models with only the actual metallicity different. The grid was normalized to pass through a hydrogen density of $n_{\mathrm{H}}=10^{11} \mathrm{~cm}^{-3}$ at $Z=5$ $Z_{\odot}$. Thermal line widths were assumed, and the flux was $\Phi\left(\mathrm{He}^{+}\right)=10^{20} \mathrm{~cm}^{-2} \mathrm{~s}^{-1}$.

Figure 15 shows that the sum of the equivalent widtlis of $\mathrm{C}$ IV $\lambda 1549$ and $\mathrm{N}$ v $\lambda 1240$ remains nearly constant, since these two lines together dominate the cooling in the more ionized regions of the cloud. At high metallicities the $\mathrm{N} \mathrm{v}$ lines carry much of the total cooling since the nitrogen abundance exceeds that of carbon when $Z \gtrsim 10 Z_{\odot}$. As $Z$ increases the equivalent width of $\mathrm{He}$ II $\lambda 1640$ decreases, due to the fact that helium absorbs an ever decreasing fraction of the incident continuum, as the metals opacity increases. As a result the equivalent widths of $\mathrm{C}$ IV and $\mathrm{He}$ II decrease, and $\mathrm{N} \mathrm{v}$ increases, with metallicity. The plot also shows that the Ly $\alpha$ and $\mathrm{O}$ VI equivalent widths change too. These are in the same sense of the signs of the equivalent width correlations known to exist with luminosity (Baldwin et al. 1989).

\subsection{A Final Model of the $\mathrm{N} v$ Region}

5.3.1. Constraints on the Model

Here we decide upon a final model of the $\mathrm{N} v$ region, component B of Paper 1. Photoionization modeling can never determine a definitively correct initial set of conditions - it can only derive conditions which produce a spectrum similar to that observed. In our final model we used the following criteria to reproduce the observed $\mathrm{N}$ v/He II, $\mathrm{O}$ vi/ $\mathrm{N} \mathrm{v}, \mathrm{C}$ iv/ $\mathrm{N}$ v ratios, and $W_{\lambda}(\mathrm{N} v)$.

The $\mathrm{N} \mathrm{V}$ region is partially transparent to ionizing radiation.-The fact that the high-ionization component does not emit low-ionization lines forced us to consider matter-bounded clouds, with the column density of the high-ionization region set by the requirement that a set of unobserved low-ionization lines not be over produced. This 
is really an observational result, independent of the modeling.

We used the lowest metallicity possible, $Z \approx 5 Z_{\odot} \cdot-$ In our models we could have relaxed other properties (i.e., the shape of the ionizing continuum) had we allowed higher metallicities. $Z \approx 5 Z_{\odot}$ can fit the spectrum only if the continuum striking the cloud between 50 and $100 \mathrm{eV}$ is dominated by the power-law component (see our discussion of the X-ray continuum below). Our result is independent of our line deconvolution. Component $\mathrm{B}$ is assigned only half of the total $\mathrm{N} v$ flux, while we take the full $\mathrm{He}$ II limit for the ratio in this region. We would have needed roughly twice as high a metallicity had the $\mathrm{He} \mathrm{II} / \mathrm{N} \mathrm{V}$ ratio been taken from the total line intensities (Table 2).

We fit the observed $U V$ continuum.-The observed continuum in $0207-398$, between rest frame wavelengths of $2000 \AA$ and $1200 \AA$, is well fitted by a power-law continuum with $f_{v} \propto$ constant $\times \exp \left(-h v / k T_{\text {cutoff }}\right)$ and an exponential cutoff corresponding to $T_{\text {cutoff }}=250,000 \mathrm{~K}$. Results are not sensitive to this slope, but they are sensitive to the cutoff temperature. The lack of signature curvature in the UV, and the requirement that the bump not extend into the $\mathrm{X}$-rays (not known for $0207-398$, but a general property of AGNs) limits the cutoff temperature to between 200,000 and $600,000 \mathrm{~K}$.

We do not understand the $X$-ray continuum.-The ratio of $\mathrm{X}$-ray to UV continua $\alpha_{o x}$ is the remaining continuum shape parameter. The observed $\alpha_{\text {ox }}$ in $0207-398$ varied between -1.57 and -1.84 between 1979 and 1992. This corresponds to a factor of of 6 change in the X-ray/UV ratio. Optical observations were obtained in 1980 and 1990, and there were no major changes in the UV line spectrum. There are two major puzzles: $(a)$ If the gas sees the same continuum we do, and the power-law continuum ionizing the $\mathrm{N} v$ region is the extrapolated X-ray continuum, then the equivalent widths of the high-ionization lines should have changed by a factor of 6 . They did not. (b) We can match only the spectrum with a metallicity as low as 5 if $\alpha_{o x}$ is significantly larger than either of the observed values. The biggest problem with small $\alpha_{o x}$ is in reproducing the observed $\mathrm{N} v$ equivalent width with covering factors less than a half.

The ionization parameter is constrained to lie near $U(\mathrm{H})=0.1$. The density is more broadly constrained to lie near $n_{\mathrm{H}}=10^{11} \mathrm{~cm}^{-3}$. - The ionization parameter is set by the relative intensities of the line spectrum, as discussed in the following section. There was a range of densities, $10^{9.7}<n_{\mathrm{H}}<10^{12} \mathrm{~cm}^{-3}$, which could reproduce the spectrum. The sharpest constraints on the density comes from the equivalent width of $\mathrm{N} v$ and the absence of detectable high-ionization lines such as $\mathrm{S}$ vi 2933 . A $\mathrm{N}$ v region covering factor of $\frac{1}{2}$ corresponds to this range of density. A more realistic covering factor of $\frac{1}{5}$ sets the density near the peak equivalent width of $10^{11} \mathrm{~cm}^{-3}$.

\subsubsection{An Optimized Model}

We produced the results presented in Table 5 by using the Subplex multidimensional optimization method developed by Rowan (1990) to find a best fit to the observed spectrum. The optimization considered various fluxes of helium ionizing photons, column densities, and hydrogen densities. We held the metallicity constant at $5 Z_{\odot}$, the smallest value capable of matching the limit to $\mathrm{N} \mathrm{v} / \mathrm{He}$ II. A continuum with a bump temperature of $250,000 \mathrm{~K}$ and
TABLE 5

PREDICTED INTENSITIES FOR COMPONENT B

\begin{tabular}{|c|c|c|}
\hline Line & I (Observed) ${ }^{\mathbf{a}}$ & I (Predicted $)^{b}$ \\
\hline C III 977 & $<0.22$ & 0.013 \\
\hline $\mathrm{N}$ III $990 \ldots \ldots \ldots$ & $<0.22$ & 0.012 \\
\hline O vi $1035 \ldots \ldots$ & 0.94 & 0.75 \\
\hline $\operatorname{Ly} \alpha 1216 \ldots \ldots \ldots$ & $<2.5$ & 0.33 \\
\hline O v] $1218 \ldots \ldots$ & $<2.5$ & 0.46 \\
\hline$N \vee 1240 \ldots \ldots \ldots$ & 1.00 & 1.00 \\
\hline Si Iv $1397 \ldots \ldots \ldots$ & $<0.22$ & 0.031 \\
\hline$O$ vi] $1402 \ldots \ldots$ & $<0.22$ & 0.22 \\
\hline$N$ IV] $1486 \ldots \ldots$ & $<0.06$ & 0.057 \\
\hline C IV $1549 \ldots \ldots$. & 0.90 & 0.72 \\
\hline He II $1640 \ldots \ldots$ & $<0.1$ & 0.068 \\
\hline $\mathrm{O}_{\mathrm{III}]} 1666 \ldots \ldots$ & $<0.10$ & 0.0041 \\
\hline $\mathrm{N}$ III] $1750 \ldots \ldots$ & $\leq 0.10$ & 0.0014 \\
\hline Al III $1860 \ldots \ldots$ & $<0.02$ & 0.0015 \\
\hline Si III] $1895 \ldots \ldots$ & $<0.02$ & 0.0006 \\
\hline C III] $1909 \ldots \ldots$ & $<0.14$ & 0.0005 \\
\hline$W_{\lambda}(\mathrm{N} v) \ldots \ldots \ldots$ & $9.7 \AA$ & $53.8 \AA$ \\
\hline
\end{tabular}

a Best estimate of intensity relative to $\mathrm{N} \vee \lambda 1240$ from Table 3.

b Predicted intensity relative to $\mathrm{N} v \lambda 1240$. The metallicity is $5 Z_{\odot}$, and the continuum described in the text is used. The density is $n_{\mathrm{H}}=10^{11.18} \mathrm{~cm}^{-3}$, the flux of helium-ionizing photons is $\Phi\left(\mathrm{He}^{+}\right)=10^{19.68} \mathrm{~cm}^{-2}$ $\mathrm{s}^{-1}$, the cloud has a column density of $4.09 \times 10^{20}$ $\mathrm{cm}^{-2}$, and the hydrogen ionization parameter is 0.1168 . The predicted $W_{\lambda}(\mathrm{N}$ v) will allow covering factors as small as 0.18 .

$\alpha_{\mathrm{ox}}=-1.2$ was assumed and not varied. These were in the best-fitting regions of Figures 13 and 14. Larger values of $\alpha_{o x}$ are preferred since these produce larger equivalent widths for $\mathrm{N} v$. Figure 12 shows that smaller ionization parameters would be required in the optimized model to match the $\mathrm{O} v \mathrm{vI} / \mathrm{N} v$ and $\mathrm{C}$ IV/N v ratios. Smaller ionization parameters produce still smaller equivalent widths, therefore larger values of $\alpha_{\text {ox }}$ were needed to accommodate the smaller equivalent width. The overall fit is within the observational uncertainties.

\subsubsection{The Transmitted Continuum}

What would we observe if we viewed the continuum source through component B? Figure 5 shows the continuum transmitted through our model. Photoelectric opacity has significantly attenuated the region between roughly 54 $\mathrm{eV}$ and $800 \mathrm{eV}$. X-ray observations of (generally lower luminosity) AGNs do not often reveal the level of soft X-ray absorption predicted in this figure, suggesting that most $\mathrm{X}$-ray lines of sight do not pass through regions with properties similar to what we have modeled. However, Voit, Weymann, \& Korista (1993) find that this type of extinction is required to produce the absorption lines in BALQSOs.

\subsection{A Relationship to BALQSOs?}

Is there any relation between the high-ionization region we model, and the absorbing gas detected in BALQSOs? The levels of ionization and column densities are similar, and it has long been known that the scattered continuum from the absorption troughs could be detected in emission when viewed from other angles. It is thought that for BALQSOs the absorbing gas is unrelated to the emission line regions and must lie outside the BLR, because absorption troughs cut across $\mathrm{Ly} \alpha$. This argument is very geometry dependent, however, since BLR clouds emit 
essentially $100 \%$ of their Ly $\alpha$ flux from the illuminated face, back into the direction of the central object (Ferland \& Netzer 1979). In this case the observed Ly $\alpha$ must be produced on the far side of the BLR - and the absorbing gas can lie anywhere along the line of sight.

We do not pursue this analogy at length here. Our calculations assume only thermal line broadening, while BALQSO clouds are thought to be an outflowing wind (Turnshek 1988). Were we to have assumed large line widths, continuum fluorescence (and $\mathrm{N} v$ scattering of broad-lined $\mathrm{Ly} \alpha$ ) would have been large too (Hamann, Korista, \& Morris 1993). Calculations show that this process does strengthen the entire emission-line spectrum, but that the relative intensities do not change greatly.

If the BALR is a disk wind with an opening angle corresponding to a covering factor of 0.1 , the opening half-angle would be $\sim 6^{\circ}$ (from eq. [9b] in Hamann et al. 1993). In order for the Ly $\alpha$-scattering gas, which is outflowing at $\sim 6000 \mathrm{~km} \mathrm{~s}^{-1}$, to scatter that flux into a profile whose FWHM is $\sim 3900 \mathrm{~km} \mathrm{~s}^{-1}$ the outflow cannot deviate more than $\arcsin (1950 / 6000=0.325)=19^{\circ}$ from the plane of the sky. Therefore, requiring a narrow scattering profile means that this idealized disk wind cannot be viewed more than $19^{\circ}-6^{\circ}=13^{\circ}$ from pole-on. For randomly oriented QSOs, the chance of viewing this geometry within $13^{\circ}$ of pole-on is only $1-\cos (13) \sim 2.5 \%$.

Calculations (Hamann \& Korista 1995) show that equivalent width of the $\mathrm{N} v$ produced in the BAL would be $84 \AA$ times the gas covering factor, or roughly $8 \AA$ for $10 \%$ coverage. This is less than the observed equivalent width of $21 \AA$, although close enough to be potentially significant. This upper limit is important only if most of the flux scatters into the narrow $\mathrm{N} v$ emission core. The scattering gas would have to lie very nearly (and exclusively) in the plane of the sky. In contrast, a spherical distribution of clouds scattering isotropically would spread the scattered flux across more than $\pm 5900 \mathrm{~km} \mathrm{~s}^{-1}$, much more than the width of the line. We conclude that scattering of $\mathrm{N} v$ by a possible BAL component cannot account for more than $\approx 10 \%$ of the measured line intensity.

\section{CONCLUSIONS}

We have considered the implications of line observations of the luminous quasar Q0207-398 for the metallicity and geometry of the ionization regions. This object is important because we can separate the emission-line regions into components, and then derive the intensities of the high- ionization region by itself. In this object profile differences play a role similar to reverberation mapping of the BLR in low-luminosity Seyfert galaxies. Line profiles separate gas into kinematic components, while the reverberation mapping separates it into geometric regions.

We concentrate on the $\mathrm{N} v \lambda 1240 / \mathrm{He}$ II $\lambda 1640$ emissionline ratio since this will indicate metallicities at or below the actual value for any geometry. This is because $\mathrm{He}$ II lines must form where $\mathrm{N} v$ emits, but the He II-emitting region need not be filled with $\mathrm{N}$ v-emitting gas. It is not possible for optically thin or fragmentary components of clouds to cause this line ratio to overestimate the $\mathrm{N} / \mathrm{He}$ ratio.

Using the observed $\mathrm{N} \mathrm{v/He}$ II line ratio as our guide, we have varied all cloud and continuum shape parameters to produce the largest possible $\mathrm{N} v / \mathrm{He}$ II ratio and $\mathrm{N} v$ equivalent width. We just succeed with a metallicity of $5 Z_{\odot}$. If the quasar continuum and cloud parameters are not similarly tuned, then an even larger metallicity will be needed to reproduce the observed limit to $\mathrm{N}$ V/He II. Even the extreme assumption of collisonal equilibrium rather than photoionization equilibrium does not alter the conclusion that solar abundances cannot reproduce the observed spectrum.

A major result is that high-ionization gas must be partially transparent to ionizing radiation. This is really an observation-little $\mathrm{N}$ IV] or $\mathrm{N}$ III] emission comes from the region making the $\mathrm{N} v$. In our calculations the $\mathrm{N} v$ region is truncated at the point where the lower ionization lines exceed their observed limits. For our final model this corresponds to a column density of $4 \times 10^{20} \mathrm{~cm}^{-2}$. We do not go into the question why the emission-line regions of quasars are segregated into high- and low-ionization regions.

Our conclusion that high-luminosity quasars are metalrich, and previous indications that this metallicity scales with luminosity, may have implications for the equivalent width-luminosity relation (Baldwin 1977). Calculations presented here show that, for metallicity above solar, the equivalent widths of $\mathrm{C} \mathrm{IV}, \mathrm{O}$ VI, and $\mathrm{He}$ II go down and that of $\mathrm{N}$ $\mathrm{V}$ goes up, as the metallicity increases. These correlations are in the sense of the observations (Osmer et al. 1994; Zheng, Kriss, \& Davidson 1995).

J. A. B. and G. J. F. thank STScI for support through GO 2306. G. J. F. and K. T. K. acknowledge the support of NSF (AST 93-19034) and NASA (NAGW-3315), and G. J. F. the Cerro Tololo Inter-American Observatory for its hospitality. F. H. was supported by NASA grant NAG 5-1630. We thank the referee, Luc Binette, for his comments.
Arimoto, N., \& Yoshii, Y. 1986, A\&A, 164, 260

1987, A\&A, 173, 23

Avni, Y., \& Tananbaum, H. 1986, ApJ, 305, 83

Baldwin, J. A. 1977, ApJ, 214, 769

Baldwin, J. A., et al. 1996, ApJ, 461, 664 (Paper I)

Baldwin, J. A., \& Netzer, H. 1978, ApJ, 226, 1

Baldwin, J. A., Wampler, E. J., \& Gaskell, C. M. 1989, ApJ, 338, 630

Barvainis, R. 1990, ApJ, 353, 419

1992, ApJ, 400, 502

Bechtold, J., et al. 1995, AJ, 108, 374

Begelman, M. C. 1989, in IAU Symp. 134, Active Galactic Nuclei, D. E.

Osterbrock \& J. S. Miller (Kluwer: Dordrecht), 141

Bica, E. 1988, A\&A, 195, 76

Bica, E., Arimoto, N., \& Alloin, D. 1988, A\&A, 202, 8

Binette, L., Prieto, A., Szuszkiewicz, E., \& Zheng, W. 1989, ApJ, 343, 135

Clavel, J., et al. 1991, ApJ, 366, 64

Collin-Souffrin, S. 1991, A\&A, 248, 389

Dalgarno, A., \& McCray, R. A. 1973, ApJ, 181, 95

Davidson, K., \& Netzer, H. 1979, Rep. Prog. Phys., 51, 715

Elitzur, M., \& Ferland, G. J. 1986, ApJ, 305, 35

\section{REFERENCES}

Ferland, G. J. 1995, Hazy, a Brief Introduction to Cloudy, Univ. Kentucky Phys. Dep. Int. Rep

Ferland, G. J., \& Netzer, H. 1979, ApJ, 229, 274

Ferland, G. J., Peterson, B. M., Horne, K., Welsh, W. F., \& Nahar, S. N. 1992 ApJ 387,95

Grevesse, N., \& Anders, E. 1989, Cosmic Abundances of Matter (AIP Conf. Proc. 183), ed. C. J. Waddington, 1

Guilbert, P., \& Rees, M. J. 1988, MNRAS, 233, 475

Hamann, F. \& Ferland, G. 1992, ApJ, 391, L53

1993, ApJ, 418, 11 (HF 93)

Hamann, F., \& Korista, K. 1995, ApJ submitted

Hamann, F., Korista, K., \& Morris, S. 1993, ApJ, 415, 541

Hummer, D. G., \& Storey, P. 1987, MNRAS, 224, 801

Kallman, T. R., Wilkes, B. J., Krolick, J. H., \& Green, R. 1993, ApJ, 403, 45

Krolik, J. H., Horne, K., Kallman, T., Malkan, M., Edelson, R., \& Kriss, G. 1991, ApJ, 371, 541

Krolik, J., McKee, C. M., \& Tarter, C. B. 1981, ApJ, 249, 422

La Franca, F., Franceschini, A., Cristiani, S., \& Vio, R. 1995, A\&A, 299, 19

Laor, A., \& Netzer, H. 1989, MNRAS, 238, 897

Lightman, A. P., \& White, T. R. 1988, ApJ, 335, 57 
Malkan, M., \& Sargent, W. L. W. 1982, ApJ, 254, 22

Mathews, W. G. 1992, ApJ, 386, 90

io. Mathews, W. G., \& Ferland, G. J. 1987, ApJ, 323, 456

Matteucci, F., \& Greggio, A. 1986, A\&A, 154, 279

Matteucci, F., \& Tornambe, A. 1987, A\&A, 185, 51

IncWilliam, A., \& Rich, R. M. 1994, ApJS, 91, 749

Mendoza, C. 1983, in IAU Symp. 103, Planetary Nebulae, ed. D. R. Flower

(Dordrecht: Reidel), 143

IOI Netzer, H. 1987, MNRAS, 225, 55

II. Osmer, P. S., Porter, A. C., \& Green, R. F. 1994, ApJ, 436, 678

Osterbrock, D. E. 1989, Astrophysics of Gaseous Nebulae and Active Galactic Nuclei (Mill Valley: University Science Press)

Rees, M. J. 1987, MNRAS, 228, 47P

Rich, R. M. 1988, AJ, 95, 828

Rokaki, E., Collin-Souffrin, S., \& Magnan, C. 1993, A\&A, 272, 8

Rokaki, E., \& Magnan, C. 1992, A\&A, 261, 41
Rowan, T. 1990, Ph.D. thesis, Univ. Texas at Austin

Sanders, D. B., et al. 1989, ApJ, 347, 29

Scalo, J. M. 1990, in Windows on Galaxies, ed. G. Fabbiano, J. S. Gallagher, \& A. Renzini (Dordrecht: Kluwer), 125

Sheilds, G. A. 1976, ApJ, 204, 330

Smith, D. A., Done, C., \& Pounds, K. E. 1993, MNRAS, 263, 54

Turnshek, D. 1988, in Space Telescope Science Inst. Symp. No. 2, QSO Absorption Lines: Probing the Universe, ed. S. C. Blades, C. Norman, \& D. Turnshek (Cambridge: Cambridge Univ. Press), 17

Uomoto, A. 1984, ApJ, 284, 497

Voit, M., Korista, K., \& Weymann, R. 1993, ApJ, 413, 95

Wilkes, B. J., \& Elvis, M. 1987, ApJ, 323, 243

Wilkes, B., et al. 1994, ApJS, 92, 53

Zamorani, G., et al. 1981, ApJ, 245, 357

Zheng, W., Kriss, G. A., \& Davidson, A. F. 1995, ApJ, 440, 606 